\title{
氢键活化的硝基烯参与的多组分不对称串联合成研究进展
}

\author{
严丽君 $a$ 闫金金 $a$ 陈雪冰*, $b$ 王永超*, $a$ \\ ( ${ }^{a}$ 云南师范大学职业技术教育学院 昆明 650092) \\ ( ${ }^{b}$ 红河学院理学院 云南蒙自 661199)
}

\begin{abstract}
摘要 有机催化多组分不对称串联反应是构建复杂手性化合物的最有效方法之一, 双功能手性催化剂是一类重要的单 分子双活化有机小分子催化剂，能同时对多个反应底物进行氢键活化，实现多个新键的形成和多个手性中心的立体选 择性控制. 基于双功能催化剂氢键活化的硝基烯是一类重要的有机反应合成子, 能参与多种有机小分子催化的串联反 应. 对硝基烯参与的多组分不对称串联反应，根据双功能催化剂的结构特征，从双功能硫嫝一胺催化、双功能方酰胺胺催化、其它双功能催化剂催化三个方面进行文献综述. 从反应类型、反应机理、反应特点及应用等方面进行了系统 地阐述, 并对该领域的研究应用和发展前景进行了展望.
\end{abstract}

关键词 双功能催化剂; 氢键活化; 硝基烯; 多组分反应; 不对称合成; 串联反应

\section{Advances in Multicomponent Asymmetric Cascade Synthesis Involving Hydrogen-Bond-Activated Nitroolefins}

\author{
Yan, Lijun ${ }^{a} \quad$ Yan, Yuxin $^{b} \quad$ Chen, Xuebing ${ }^{*, b} \quad$ Wang, Yongchao ${ }^{*, a}$ \\ ( ${ }^{a}$ College of Vocational and Technical Education, Yunnan Normal University, Kunming 650092) \\ ( ${ }^{b}$ School of Science, Honghe University, Mengzi, Yunnan 6611992)
}

\begin{abstract}
Organocatalytic multicomponent asymmetric cascade reaction is generally regarded as one of the most effective methods for constructing complex chiral compounds. Bifunctional chiral catalysts are an important class of single-molecule double-activated organic catalysts, which can simultaneously activate hydrogen bonds of multiple reactive substrates to achieve the formation of multiple new bonds and stereoselective control of multiple chiral centers. Nitroolefins are important organic reaction synthons, which can participate in a variety of asymmetric cascade reactions by hydrogen bond activation. In this paper, the recent advances in nitroolefins-involved multicomponent asymmetric cascade reactions catalyzed by bifunctional organocatalysts involving chiral bifunctional thiourea amines, squaramide amines and other bifunctional catalysts are reviewed. Specifically, the catalytic systems, characteristics, mechanisms are systematically expounded, and the application of this research field is also prospected.
\end{abstract}

Keywords bifunctional catalysts; hydrogen-bond activation; nitroolefin; multicomponent reactions; asymmetric synthesis; cascade reaction

有机催化多组分不对称串联反应是构建复杂手性 化合物的最有效方法之一，在有机合成领域中占据重要 地位 ${ }^{[1]}$. 双功能手性小分子催化剂存在两个催化活性位 点, 可通过氢键作用，协同酸催化、碱催化等模式，同时 对多个底物进行活化. 基于双功能催化剂氢键活化的多 组分不对称串联反应具有高效、操作简洁、后处理简单、 立体选择性优异等优点, 具有重要的研究开发价值 ${ }^{[2]}$.
1981 年, Hiemstra 和 Wynberg ${ }^{[3]}$ 首次提出了双功能 催化剂的概念, 并发现双功能奎宁能通过氢键对反应进 行立体选择性控制. Okino 等 ${ }^{[4]}$ 于 2003 年开创性地提出 了单分子双活化的概念, 并首次合成了双功能硫䏱-胺 催化剂 1a，该催化剂在硝基烯与丙二酸酯的不对称 Michael 加成反应中表现出良好的催化活性(Eq. 1). 随 后的十余年间，大量的双功能手性催化剂相继被合成和

\footnotetext{
* Corresponding authors. E-mail: yongchaowang126@126.com; orangekaka@126.com Received October 31, 2019; revised December 2, 2019; published online December 19, 2019.

Project supported by the Applied Basic Research Project Foundation of Yunnan Provincial Department of Science and Technology (Nos. 2018FD016, 2017FD073, 2017FD156).

云南省科技厅应用基础研究(Nos. 2018FD016, 2017FD073, 2017FD156)资助项目.
} 
应用, 取得了重大进展 ${ }^{[5]}$. 目前研究得比较多的双功能 有机催化剂主要集中在硫艮-胺类、方酰胺-胺类、联二 菜酚(BINOL)-手性磷酸类及其它类型. 这些催化剂的主 要催化作用机制是基于氢键对反应底物进行活化, 从而 实现对反应的手性控制. 硝基烯烃是一类重要的有机合 成子[6], 双功能有机催化剂通过活性基团( $\mathrm{NH} 、 \mathrm{OH}$ 等) 与硝基烯烃的硝基形成双氢键、可以活化硝基的 $\alpha-\mathrm{C}$, 同时还可以通过胺基、磷酰基等基团对其它底物(或中 间体)的活性基团进行选择性活化，从而控制反应的非 对映异构体选择性和对映异构体选择性.

$$
\mathrm{R}_{2}^{\mathrm{NO}_{2}}+\mathrm{R}^{2}
$$

up to $88 \%$ yield up to $93 \%$ ee

$$
\mathrm{R}^{2} \mathrm{O}_{2} \mathrm{C}{ }_{3 a}^{\mathrm{R}^{3}} \mathrm{CO}_{2} \mathrm{R}^{2} \stackrel{1 \mathbf{a}(10 \mathrm{~mol} \%)}{\text { Toluene, r.t. }}
$$

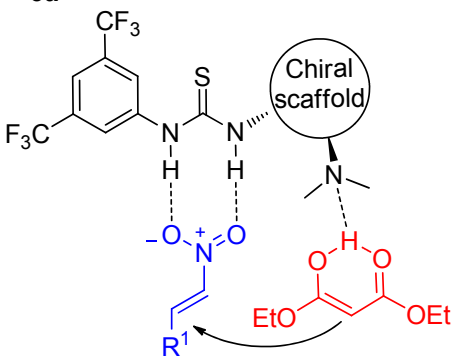

Double hydrogen-bonds activation mechanism
近年来, 基于双功能催化剂氢键活化的硝基烯参与
的多组分不对称串联合成研究受到了国内外化学及药 学家的普遍关注，并取得了重大进展. 本文根据硝基烯 烃参与的多组分不对称串联反应体系的双功能催化剂 类型, 分别从双功能硫脲-胺催化、双功能方酰胺-胺催 化、其它双功能催化剂催化三个方面进行文献综述，对 反应的催化体系、反应的主要特点、催化机理等方面进 行系统阐述, 并对多组分不对称串联合成的应用研究进 行了展望.

\section{1 双功能硫腿-胺催化的多组分不对称串联反 应研究}

Sigman 等 ${ }^{[7]}$ 早在 1998 年就发现手性硫脲衍生物可 以有效地催化 Strecker 等多种反应，但直到 2003 年 Okino 等 ${ }^{[4]}$ 才报道了首例双功能硫嫝-胺催化剂的合成 及应用, 并开创性地提出了单分子双活化的概念. 随后 十余年间, 大量的双功能硫脲一胺催化剂(1)相继被合成 和应用. 双功能硫嫝一胺催化剂含有两个特征性的仲胺 基团，可作为双氢键供体，并含有一个或两个硫艮基， 可以同时活化亲电试剂与亲核试剂. 大多数双功能硫脲 催化剂还包含手性环己二胺、奎宁(金鸡纳碱)、手性乙 二胺等骨架, 在多组分不对称串联反应中表现出突出的 催化活性.<smiles>CN(C)[C@@H]1CCCC[C@H]1NC(=S)Nc1cc(C(F)(F)F)cc(C(F)(F)F)c1</smiles><smiles>C=CC1=CC[C@@H]([C@H](Cc2ccccc2)c2ccnc3ccc(NC(=S)NC[C@H](NS)C(C)OC(C)(C)C)cc23)NC1</smiles>

$1 e$<smiles>N[C@@H]1CCCC[C@H]1NC(=S)Nc1cc(C(F)(F)F)cc(C(F)(F)F)c1</smiles>

1b

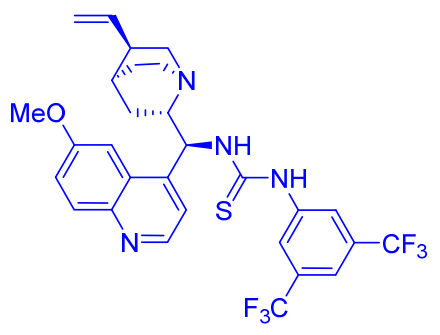

$1 c$

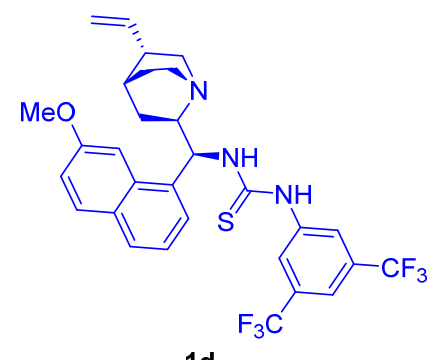

1d

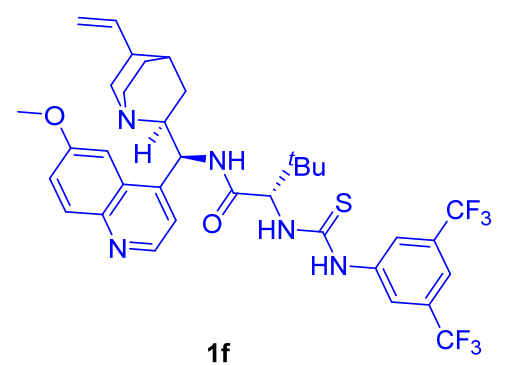<smiles>CC([C@H](NC(=S)Nc1cc(C(F)(F)F)cc(C(F)(F)F)c1)c1ccccc1)N1CCCCC1</smiles>

$1 \mathbf{i}$<smiles>CCC1=CNC(C(NC(=S)Nc2ccc(C(F)(F)F)cc2)c2cccc3ccc(OC)cc23)CC1</smiles>

19<smiles>CC(C)C(CN(C)C)NC(=S)Nc1cc(C(F)(F)F)cc(C(F)(F)F)c1</smiles>

$1 \mathrm{~h}$

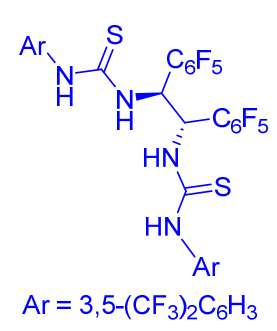

$1 \mathrm{j}$ 


\section{1 双功能硫脲-环己二胺催化的多组分不对称串联 反应}

氮杂糖是一类重要的哌啶衍生物, 具有多种药用活 性 ${ }^{[8]}$. Barbas III 等 ${ }^{[9]}$ 于 2010 年报道了基于手性双功能硫 嫝-伯胺 $1 \mathbf{b}$ 催化的三组分不对称 anti-Michael/anti-azaHenry 串联反应，合成了一系列氮杂糖衍生物 5 (Eq. 2). 在 $1 \mathbf{b}$ 催化剂、四甲基胍(TMG)为碱、 $\mathrm{AcOH}$ 为添加剂的 条件下, 硝基烯(2)和脂肪醛(6a)经双氢键活化, 发生分 子间的 anti-Michael 反应，得到的 $\gamma$-硝基酫中间体(I)进 一步与亚胺化合物(7a)发生 anti-aza-Henry 串联反应, 只 需 $0.5 \mathrm{~h}$, 以 $98 \% \sim 99 \%$ 的立体选择性 (ee 值)构建了 3 个 新的 $\mathrm{C}-\mathrm{C}$ 键和 5 个连续手性中心.

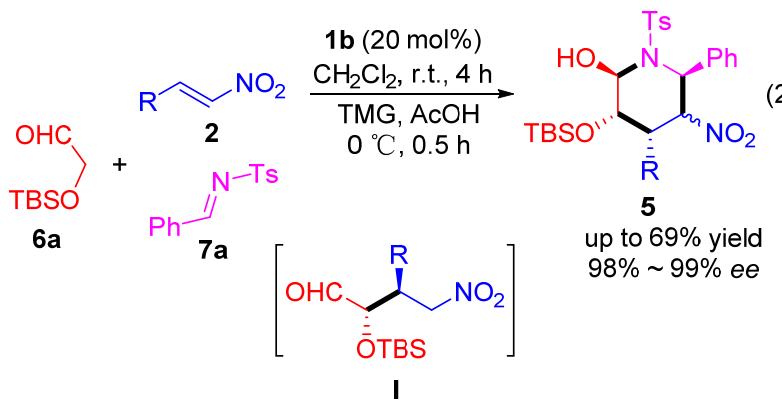

此外, Barbas III 等 ${ }^{[10]}$ 又开发了手性双功能硫脲一伯 胺 $1 \mathbf{b}$ 催化的多组分不对称 anti-Michael/Michael/Henry 串联反应，并合成了手性构型不同的氢化吡喃糖衍生物 $8 \mathbf{a}$ 和 $8 \mathbf{b}$ (Scheme 1). 反应基于脂肪醛(6a)、硝基苯乙烯 (2a)和乙醛酸乙酯(9)的三组分不对称串联反应，通过选 用不同的有机碱( $\mathrm{Et}_{3} \mathrm{~N}$ 或 $\left.\mathrm{DBU}\right)$ 进行反应, 得到了硝基 $\alpha-\mathrm{C}$ 构型翻转的手性氢化吡喃化合物 $8 \mathrm{a}$ 和 $8 \mathrm{~b}$. 该反应
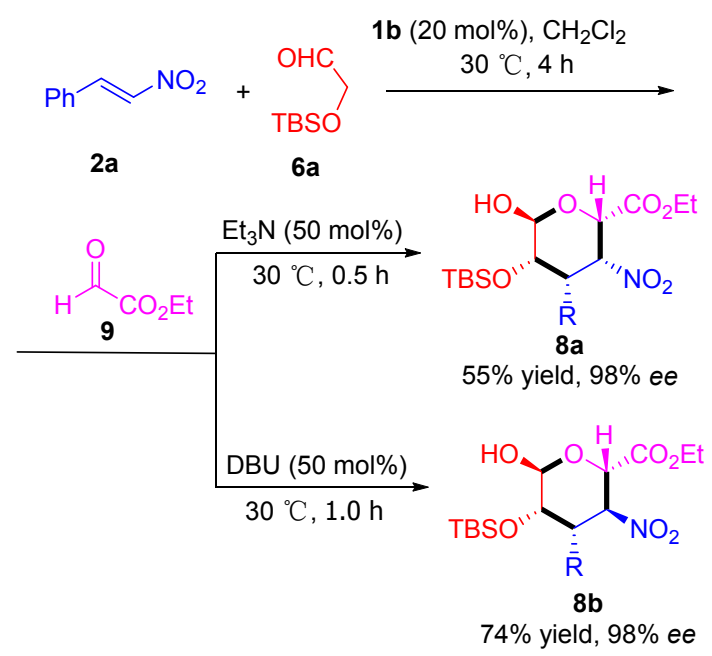

图式 1 双功能硫脲-伯胺催化的三组分不对称 anti-Michael/ anti-aza-Henry 串联反应合成氢化吡喃糖衍生物 8

Scheme 1 Synthesis of hydrogenated pyranose derivatives (8) by bifunctional thiourea-primary amine catalyzed three-component asymmetric anti-Michael/anti-aza-Henry cascade reaction
耗时短 $(0.5 \mathrm{~h}$ 和 $1.0 \mathrm{~h})$ 、立体选择性好, 但底物的普适性 研究还有待进一步考察.

Rodriguez 等 ${ }^{[11]}$ 于 2010 年建立了双功能硫脲-胺催 化的 $\alpha$-酰胺酩(10a)和两分子 4-F 硝基苯乙烯 $(2 \mathrm{~b})$ 的不对 称 Michael/Michael/Henry 串联反应(Eq. 3). 在双功能手 性催化剂 $1 \mathrm{a}$ 的双氢键作用下，发生两分子 4-F 硝基苯乙 烯(2b)和 $\alpha$-酰胺酮(10a)的双 Michael 加成, 其加成产物 再发生分子内不对称 Henry 环化反应(如 TS 所示)，以良 好的非对映异构体选择性 $(>20: 1 d r)$ 、优秀的对映异构 体选择性(98\% ee) 合成了多功能团化的环己烷衍生物 11.
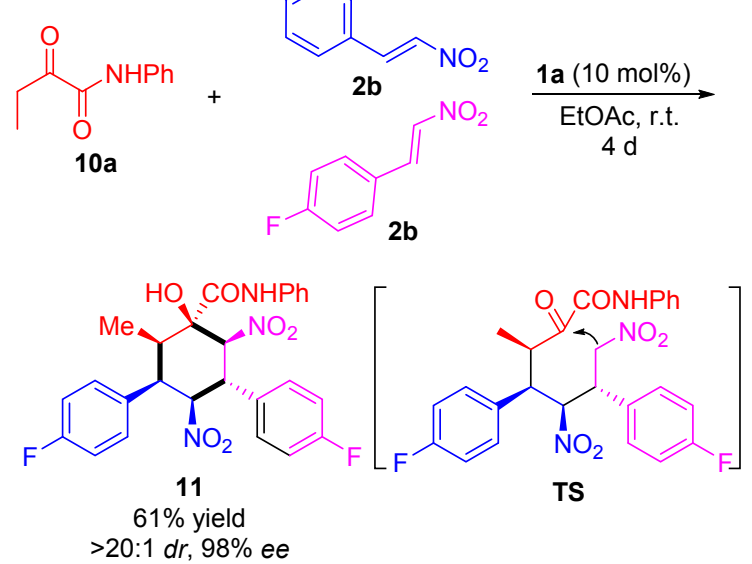

1.2 双功能硫脲一奎宁胺催化的多组分不对称串联反 应

许鹏飞课题组 ${ }^{[12]}$ 于 2009 年开发了手性双功能硫脲 -奎宁胺 1c 和手性仲胺 12 协同催化的硝基烯(2)、丙二 酸酯(3)和 $\alpha, \beta$-不饱和醛(13)的三组分不对称 Michael/ nitro-Michael/aldol 串联反应(Scheme 2). 通过双功能硫 嫝-奎宁胺 1c 对硝基的氢键活化和 Brøsted 酸/碱的双功 能催化，发生硝基烯(2)和丙二酸酯(3)的不对称 Michael 加成得到中间体 14. $\alpha, \beta$-不饱和醛(13)经手性仲胺 12 的 亚胺活化后，与中间体 14 发生不对称 nitro-Michael 反 应，得到的中间体 15 再经碱催化的分子内 Aldol 反应， 以理想的对映异构体选择性(88\% > $>99 \%$ ee ) 合成了一 系列多取代手性环已烷衍生物 16. 该方法通过使用构 型不同的手性仲胺 12a 和 $12 \mathrm{~b}$ 为协同催化剂, 实现了目 标化合物的手性构型翻转.

2012 年, 王锐课题组 ${ }^{[13]}$ 开发了脂肪醛 6 和两分子硝 基烯 2 的三组分不对称 Michael/Michael/Henry 串联反 应，在双功能手性硫嫝-奎宁胺 $1 \mathrm{c}$ 和二苯基脯氨硅醚 12c 的协同催化作用下，合成了具有六个连续立体中心 的多取代环己烷衍生物 17 (Scheme 3). 该方法首先在 12c 的催化作用下通过烯胺活化发生不对称 Michael 加 


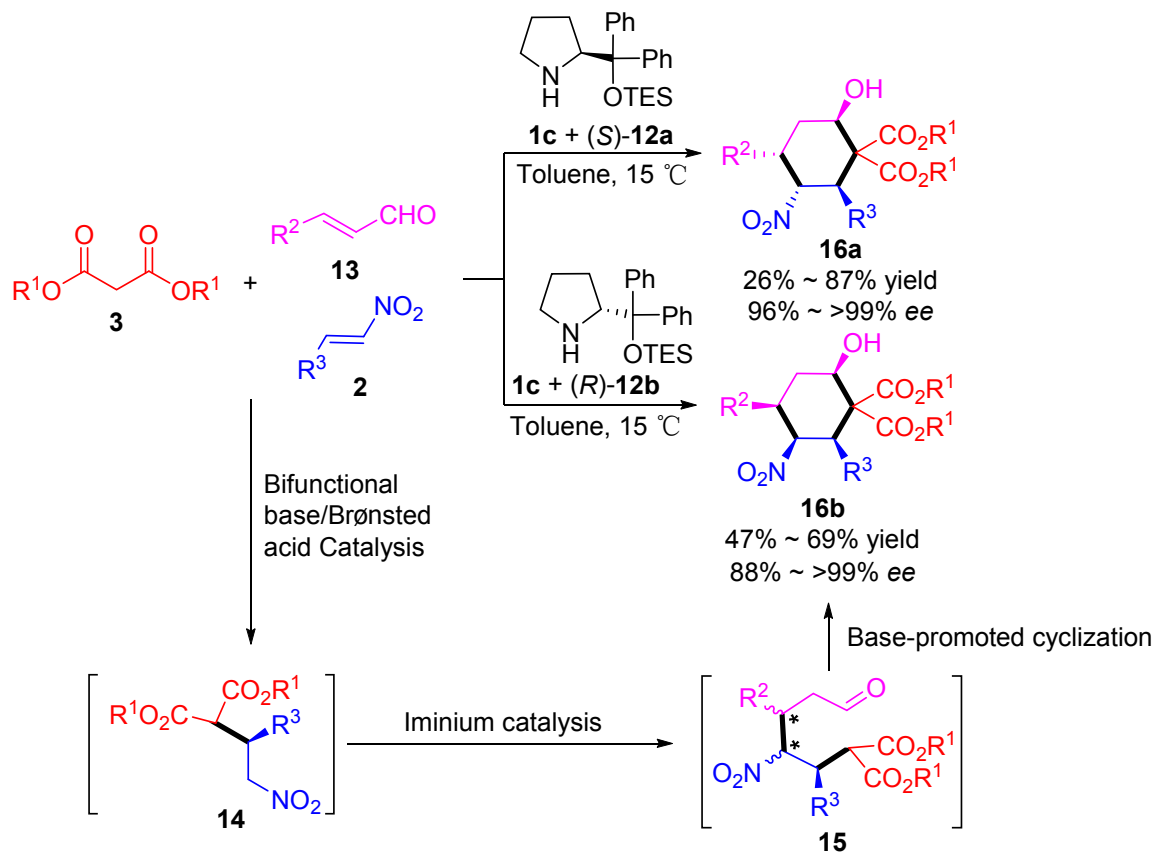

图式 2 有机催化三组分不对称 Michael/nitro-Michael/aldol 夰联反应合成手性环已烷衍生物 $\mathbf{1 6}$

Scheme 2 Asymmetric organocatalytic three-component Michael/nitro-Michael/aldol cascade reaction to synthesize chiral cyclohexane derivatives 16

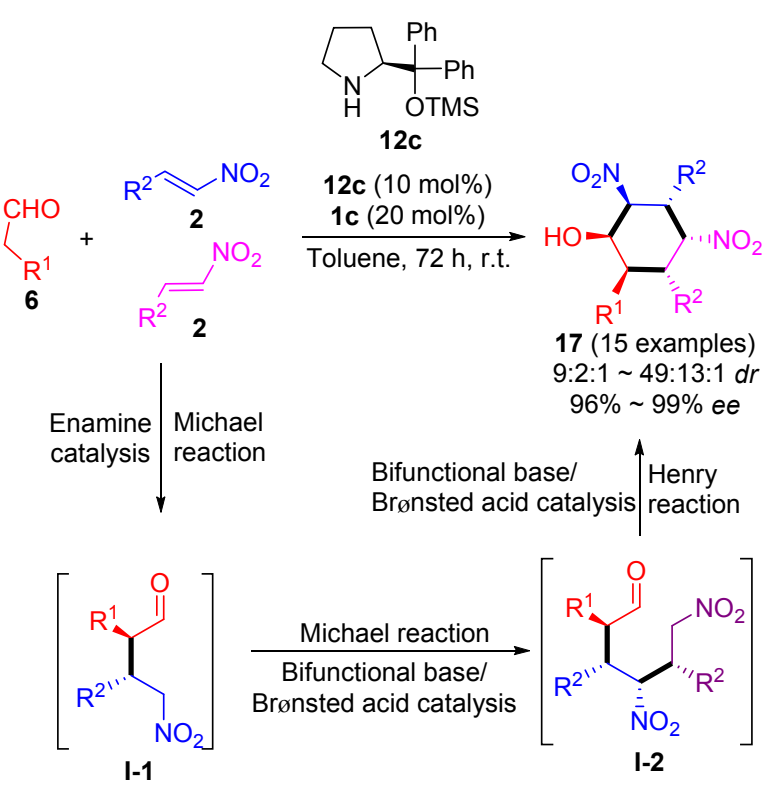

图式 3 有机催化三组分不对称 Michael/Michael/Henry 串联 反应合成全取代手性环己烷衍生物 17

Scheme 3 Asymmetric organocatalytic three-component Michael/Michael/Henry cascade reaction to synthesize chiral fully substituted cyclohexane derivatives 17

成得到中间体 I-1, 然后利用硫脲-奎宁胺 $1 \mathbf{c}$ 的氢键作用 发生二级不对称 Michael 加成得到中间体 I-2, 再经氢键 催化的不对称 Henry 反应, 以突出的非对映异构体 $(9$ : $2 ： 1 \sim 49 ： 13 ： 1 d r)$ 和对映异构体选择性(96\% 99\% $e e)$ 实现了三个新 $\mathrm{C}-\mathrm{C}$ 键的形成和六个手性中心的立体 选择性控制.
基于有机催化的化学动力学拆分技术已发展成为 制备光学纯手性分子的最有效手段之一 ${ }^{[14]} .2012$ 年, 陈 焜铭课题组 ${ }^{[15]}$ 报道了首例基于动力学拆分法的三组分 不对称串联反应(Scheme 4). 反应从吲哚(18a)、丙烯醛 (13a) 和外消旋硝基烯丙基乙酸酯(2c) 出发, 通过亚胺烯胺活化, 实现对化合物 $\mathbf{2 c}$ 进行动力学拆分, 以 $97 \% e e$ 的立体选择性获得手性吲哚衍生物 19.19 在 $20 \mathrm{~mol} \%$ 双 功能手性硫嫝-奎宁胺 1d 的催化作用下, 发生分子内亲 电一环化反应，以>99\% ee 的立体选择性得到刮哚衍生 物 20. 研究发现, 该动力学拆分可以把底物的用量增至 “克” 量级, 而不影响产物的光学纯度, 可用于规模化的 动力学拆分和高手性纯吲哚衍生物的工业化制备.

周兵等 ${ }^{[16]} 2013$ 年开发了基于双功能硫脲-奎宁 胺 $1 \mathrm{c}$ 和二苯基脯氨醇硅醚 $(12 \mathrm{c}$ 或 $12 \mathrm{~d})$ 协同催化的三组 分不对称 Michael/Michael/aldol 串联反应，合成了两类 手性 3,3'-螺环己烷氧化吲哚衍生物 21 22 (Scheme 5). 该方法以 $N$-Boc-2-氧化吲哚(23)、硝基烯化合物(2)及 $\alpha, \beta$-不饱和醛 (13)为原料, 经 “一锅法” 串联反应实现三 个新 $\mathrm{C}-\mathrm{C}$ 键、五个连续手性中心的构建. 当使用不同 的二苯基脯氨醇硅醚 12c 或 12d 协同催化时, 分别得到 了 3-螺环手性构型翻转的吲哚衍生物. 在反应中, 双功 能硫嫝一奎宁胺 $1 \mathrm{c}$ 通过氢键作用对硝基烯化合物进行活 化, 同时 1c 的叔胺结构作为 Brønsted 碱可以诱导 $N$-Boc-2-氧化吲哚(23)酰胺结构的烯醇化, 促进其与硝 基烯化合物(2)的不对称 Michael 加成(如 TS 所示). 
<smiles></smiles>

$18 \mathrm{a}$

$( \pm)-2 c$<smiles>CCOC(=O)C(OCC)/C(C)=C/c1ccccc1</smiles>

\section{2c (5 mol\%), toluene, $0{ }^{\circ} \mathrm{C}$}

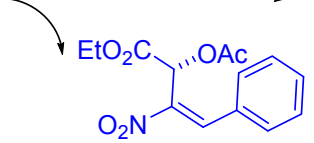

$(R)-2 \mathrm{c}$

$32 \%$ yield, $91 \%$ ee<smiles>[R]c1ccc2[nH]cc(C[C@H](CO)[C@H](C=O)C(=CC(=O)OCC)c3ccccc3)c2c1</smiles>

19

$40 \%$ yield, $97 \%$ ee

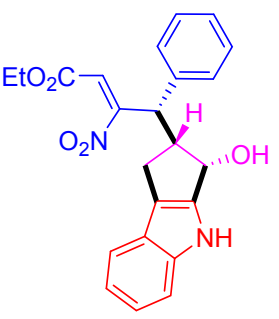

$20(40 \%$ yield, $>99 \%$ ee $)$
图式 4 有机不对称催化三组分动力学拆分合成手性吲哚衍 生物 20

Scheme 4 Asymmetric organocatalytic three-component kinetic resolution to synthesize chiral indole derivatives $\mathbf{2 0}$

手性氨基硫结构单元普遍存在于 Bestatin、Taxol、 Amastatin 等具有生物活性的分子中, 建立高效的方法 实现手性氨基硫化物的不对称合成受到化学家和药学 家的普遍关注 ${ }^{[17]} .2015$ 年, 彭云贵研究组 ${ }^{[18]}$ 开发了双功 能硫腿-奎宁胺 1e 催化的多组分不对称 sulfa-Michael/ Mannich 串联反应，合成了一系列手性氨基硫化合物 24 (Eq. 4). 该合成在手性化合物 $1 \mathrm{e}$ 的催化作用下, 首先发 生硝基烯(2)和硫酚(25)的不对称 sulfa-Michael 加成, 1e 再通过 NH 与 sulfa-Michael 加成产物的硝基形成氢键, 控制其与 $N$-Ts 烯烃(7b)的成键方向(如 TS 所示), 经不对 称 Mannich 反应得到高手性纯的化合物 24.24 可进一步 转化成具有潜在生物活性的 1,2-二氨基有机硫和 2-硝基
烯丙胺，在新药合成领域中具有潜在的应用价值.

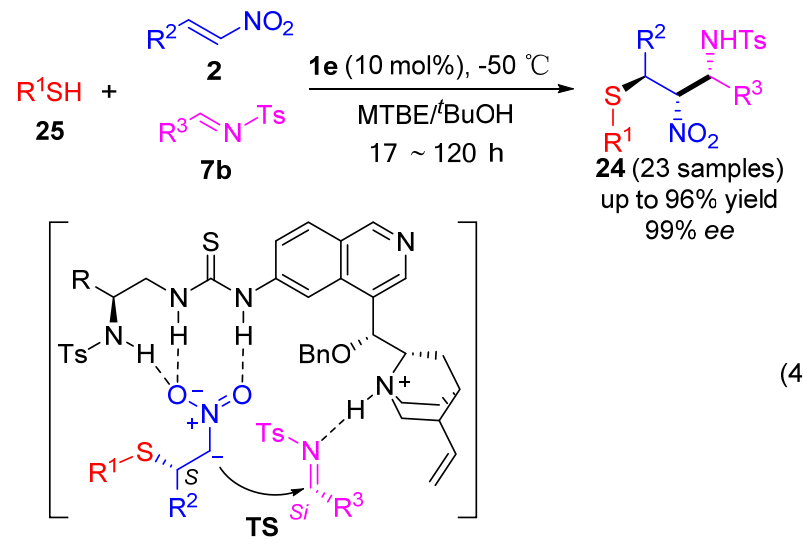

随后, 该研究组 ${ }^{[19]}$ 又开发了多功能手性硫脲一奎宁 胺 1f 在不对称 sulfa-Michael/aldol 串联反应中的催化应 用(Scheme 6). 通过氢键活化的三组分不对称串联反应, 以良好的产率(34\% 97\%)、突出的非对映异构体选择 性(90：10 >99：1 dr) 和对映异构体选择性(94\% $99 \% e e$ ) 合成了 23 个新型 $\gamma$-硫颈 $-\beta$-硝基酯(26). 化合物 26a 很容易进一步转化成具有潜在生物活性的 Bestatin 衍生物 28 和 2-硝基烯醇类化合物 29. 此外, 将反应量 放大到 “克” 量级别时，反应依然可以顺利进行，且不 影响产物的产率和立体选择性.

引入氟原子以改变药物分子的生物活性、生物利用 度和代谢特性已成为药物研究的一种普遍做法 ${ }^{[20]}$, 而 有机氟化合物的特殊作用使有机氟化，特别是不对称氟 化成为了新医药和农用化学品开发中的活跃研究课 题 ${ }^{[21]}$. 基于含氟硫脲-奎宁胺 $1 \mathrm{~g}$ 氢键活化的四组分 fluorination/Michael/Michael/aldol 串联反应于 2017 年被 研究报道 ${ }^{[22]}$ (Scheme 7). 该方法以 1,3-二羰基化合物(30) 为原料, 与 31 发生氟化反应, 得到的中间体 I-1 进一步

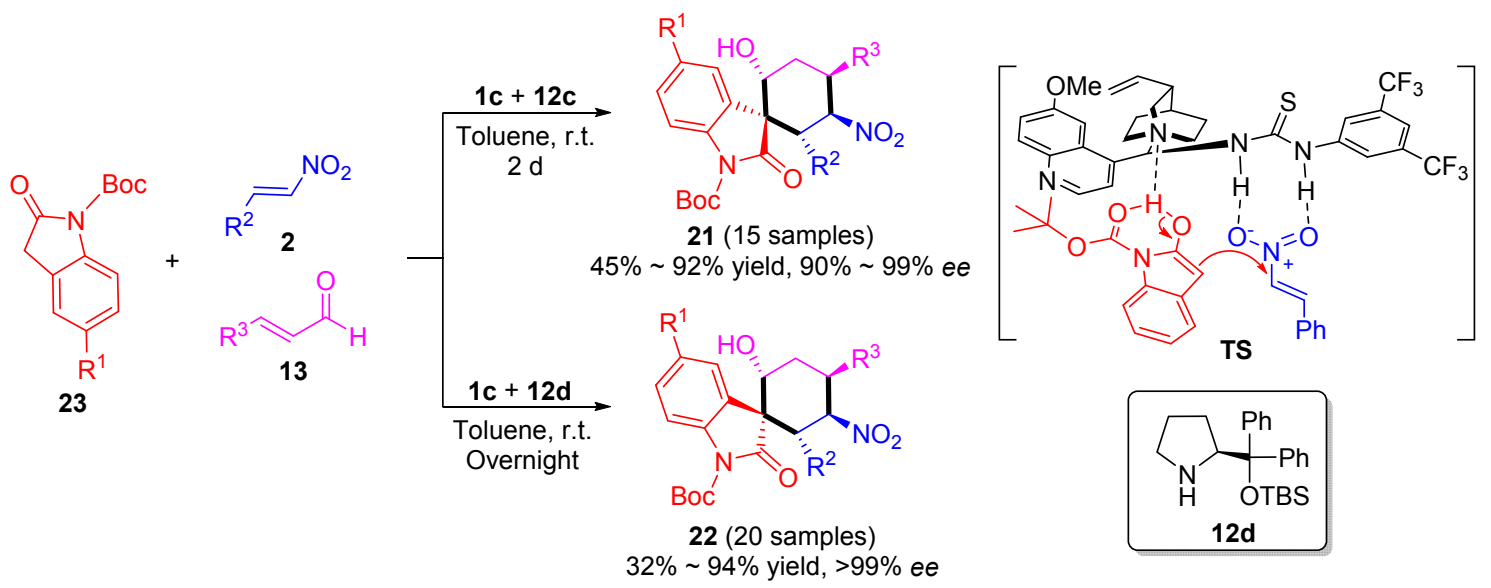

图式 5 有机催化三组分 Michael/Michael/aldol 不对称串联反应

Scheme 5 Asymmetric organocatalytic three-component Michael/Michael/aldol cascade reaction 


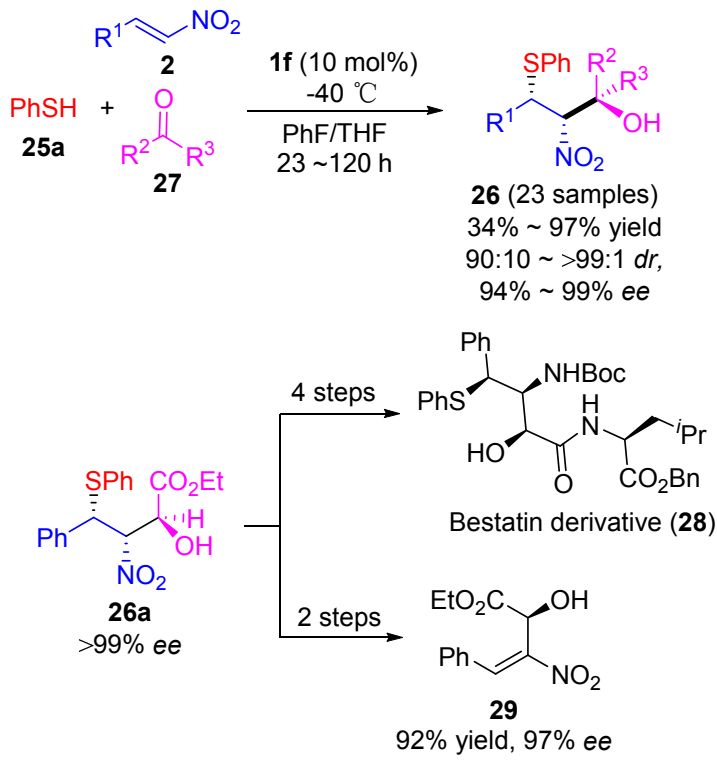

图式 6 对映选择性三组分 sulfa-Michael/aldol 串联反应及其 合成应用

Scheme 6 Enantioselective three-component sulfa-Michael/ aldol cascade reaction and its synthetic application

与硝基烯(2d)发生不对称 Michael 加成, 中间体 I-2 再与 肉桂醛(13b)发生分子间 Michael 加成, 最后发生分子内 aldol 反应，以优异的立体选择性( $>20: 1 d r, 89 \%$ 99\% ee) 合成了一系列全取代含氟手性环已烷衍生物 32.

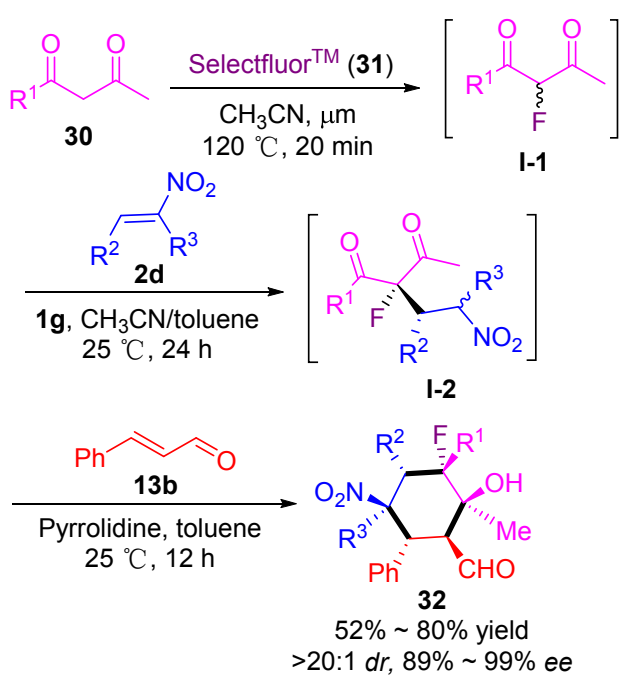

图式 7 有机催化三组分 fluorination/Michael/Michael/aldol 不 对称串联反应

Scheme 7 Asymmetric organocatalytic three-component fluorination/Michael/Michael/aldol cascade reaction

\section{3 其它双功能硫腿催化的多组分不对称串联反应}

2010 年, 许鹏飞课题组 ${ }^{[23]}$ 发展了手性双功能硫脉胺 $1 \mathrm{~h}$ 和二苯基脯氨醇硅醚(12a)协同催化的三组分不对 称 Michael/nitro-Mannich/cyclization 串联反应(Scheme
8). 通过手性仲胺 12a 的烯胺-氢键催化，发生脂肪醛(6) 和硝基烯烃(2)的不对称 Michael 加成, 得到中间体 I-1 再与 $N$-Ts 烯烃 $(7 \mathbf{b})$ 依次发生 $\mathbf{1 h}$ 催化的 nitro-Mannich 反 应和环化反应，以良好的对映异构体选择性( $98 \%$ 99\% $e e)$ 合成了一系列全取代功能团化的哌啶衍生物 33. 化 合物 33 可以进一步延伸合成吡咯烷酮、四氢吡啶等具 有潜在应用价值的手性化合物.

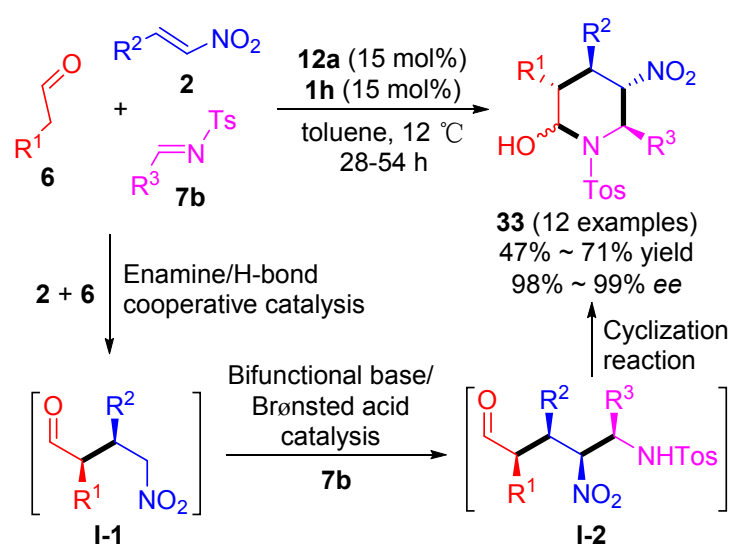

图式 8 有机催化三组分不对称 Michael/nitro-Mannich/cyclization 串联反应合成全取代手性哌啶化合物 33

Scheme 8 Asymmetric organocatalytic three-component Michael/nitro-Mannich/cyclization cascade reaction to synthesize chiral fully substituted piperidines $\mathbf{3 3}$

利用手性乙二胺衍生的双功能硫脲-胺 $1 \mathbf{i}$ 为催化剂, 基于硝基烯烃(2)、 $\beta$-酮酸酯(30a)和 $\alpha, \beta$-不饱和醛(13)的 三组分 “一锅法” 不对称串联反应被 Enders 课题组研究 报道, 并合成了一系列全取代手性环已烷衍生物 $34^{[24]}$ (Scheme 9). 该反应基于双功能催化剂氢键活化的三组 分不对称 Michael/Michael/aldol 串联反应，构建了三个 新的 $\mathrm{C}-\mathrm{C}$ 键和六个连续的立体中心(含一个手性季碳 中心), 取得了优异的立体选择性(>95: $5 d r, 91 \%$ 99\% ee). 该方法具有良好的底物普适性, 全取代环已烷 衍生物可进一步应用于天然产物及复杂手性分子的不 对称合成.

2015 年, 谭斌课题组 ${ }^{[25]}$ 合成了双功能双硫脲-胺 1f, 并建立了催化剂 $\mathbf{1 f}$ 催化的硝基烯烃(2)、重氮氧化吲哚 (35a)和亚硝基苯衍生物(36)的三组分不对称串联反应, 合成了一系列新型手性螺环氧化吲哚衍生物 37 (Scheme 10). 在反应中, 催化剂 1f 通过双重氢键作用(如 TS-1 和 TS-2 所示)对底物亚硝基苯衍生物(36)和硝基烯 烃(2)依次进行选择性氢键活化, 反应经历 $6 \mathrm{~d}$ 的室温摚 拌，以理想的产率(72\% 99\%)取得了优异的非对映异 构体选择性(>99：1 dr) 和对映异构体选择性(97\% $99 \%$ ee). 研究发现该方法对各类反应底物都表现出良 好的普适性, 并合成了 26 个高手性纯的目标化合物. 如 


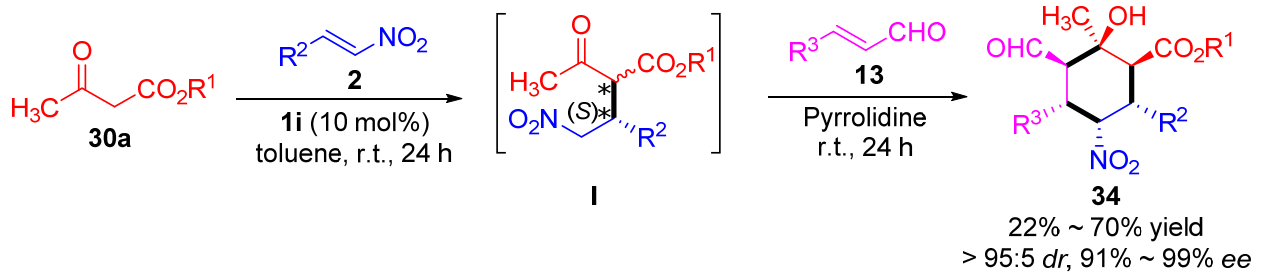

图式 9 有机催化三组分不对称 Michael/Michael/aldol 串联反应合成环已烷衍生物 34

Scheme 9 Asymmetric organocatalytic three-component Michael/Michael/aldol cascade reaction to synthesize chiral cyclohexane derivatives 34
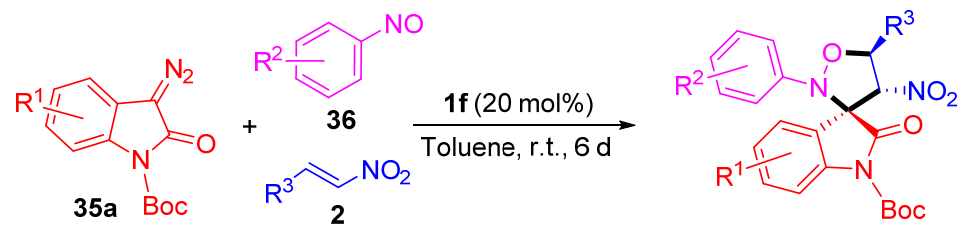

37 (26 samples)

$72 \% \sim 99 \%$ yield

$>99: 1 d r$

$97 \% \sim 99 \%$ ee
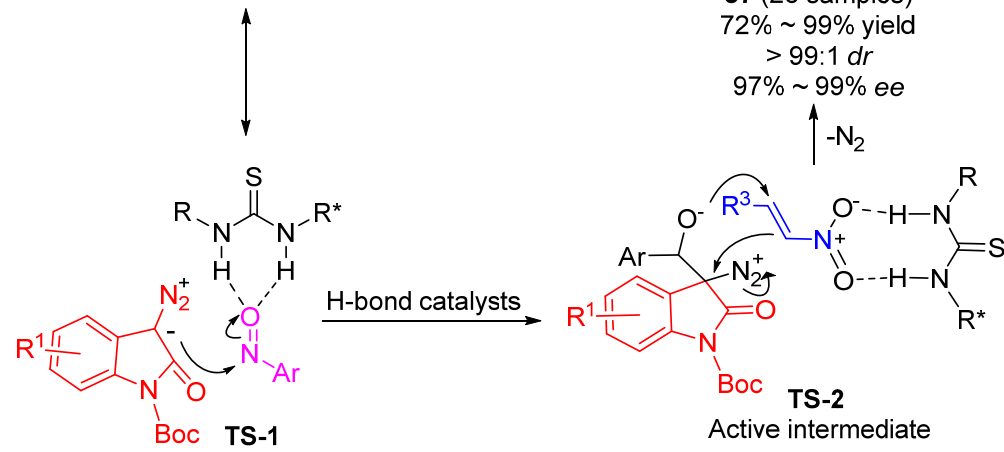

图式 10 有机催化多组分不对称串联反应合成手性螺环吲哚衍生物 $\mathbf{3 7}$

Scheme 10 Asymmetric organocatalytic cascade reaction to synthesize chiral spirooxindole derivatives 37

何提高反应速率以缩短反应时间可能是该方法的工业 化应用需要解决的一个主要科学问题.

\section{2 双功能方酰胺-胺催化的多组分不对称串联 反应}

2008 年, Malerich 等 ${ }^{[26]}$ 第一次报道了金鸡纳碱衍生 的双功能催化剂方酰胺-胺 38a 的合成, 并将其成功地 应用于二羰基化合物对硝基烯的 Michael 加成反应中, 以 $0.5 \mathrm{~mol} \%$ 的催化剂量取得了高达 $99 \%$ 的产率和 $98 \%$ $e e$ 的对映异构体选择性, 由此开辟了双功能方酰胺-胺 催化剂在有机合成反应中的应用.

双功能方酰胺手性催化剂的分子结构中存在两个 $\mathrm{NH}$ 键和两个羰基, 能与反应物的多种基团形成多氢键 作用, 是一类典型的氢键供体 ${ }^{[27]}$. 此外, 与双功能硫艮 相比，手性方酰胺氮氢键有一定的夹角，同时两个羰基 的作用使方酰胺显更强的酸性, 能更有效地与底物结合 并激活底物分子. 这些特殊的结构及性质特征使得双功 能方酰胺手性催化剂在多类多组分不对称催化串联反 应中均表现出良好的催化活性, 大量的新型双功能方酰
胺催化剂(38)也相继被开发应用 ${ }^{[28]}$.

\section{1 双功能方酰胺-奎宁胺催化的多组分不对称串联} 反应

吡咯螺环氧化吲哚衍生物具有抗菌、抗肿瘤、抗糖 尿病、抗炎、抗结核等多种药用活性，建立构建手性吡 咯螺环氧化吲哚骨架的新方法受到化学家和药学家的 普遍关注 ${ }^{[29]} .2013$ 年, 许鹏飞课题组 ${ }^{[30]}$ 建立了有机不对 称催化多组分串联反应合成手性 3,2'-吡咯烷酮螺环吲 哚 39 的新方法(Eq. 5). 利用双功能方酰胺-奎宁胺 38c 的氢键催化作用, 开展了硝基烯烃(2)、吲哚醌(40)和茮 胺(41a)的三组分不对称 1,3-质子转移和 [3+2] 环加成串 联反应，实现三个新的 $\mathrm{C}-\mathrm{C}$ 键和四个手性中心的构筑， 取得了良好的非对映异构体选择性(5：1１1：1 dr) 和 对映异构体选择性 $(77 \%$ \% $96 \%$ ee $)$.

2014 年, 龚流柱课题组 ${ }^{[31]}$ 开发了金属有机催化的 三组分不对称 C-H Functionalization/Michael 串联反应 的方法及其应用研究(Scheme 11). 利用双功能手性方酰 胺-奎宁胺 38d 和金属钓(II)或铑(II)形成的有机配合物 为催化剂, 基于氢键活化的方式(如 TS 所示)开发了硝 
<smiles>C=CC12CCC(C1)N2CC(NC1C(=O)C(=O)C1NCc1cc(C(F)(F)F)cc(C(F)(F)F)c1)c1ccnc2ccccc12</smiles><smiles>C=CC1CCCN1C(Cc1ccnc2ccccc12)Nc1c(NCc2cc(C(F)(F)F)cc(C(F)(F)F)c2)c(=O)c1=O</smiles>

$38 b$<smiles>C=CC1CCC(c2cncc3ccc(OC)cc23)C(NC2=C(Nc3cc(C(F)(F)F)cc(C(F)(F)F)c3)C(=O)OC2=O)N1</smiles>

$38 c$<smiles></smiles><smiles>C=CC1C2CCC1N(Cc1ccnc3ccc(OC)cc13)C(Cc1c(NCc3cc(C(F)(F)F)cc(C(F)(F)F)c3)c(=O)c1=O)C2</smiles>

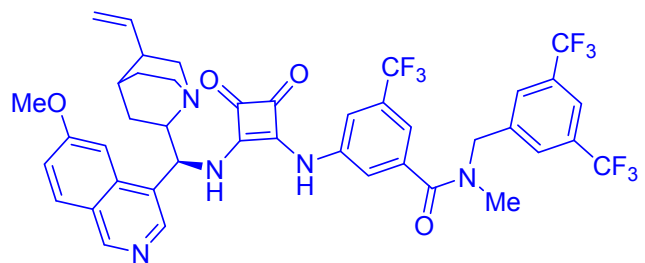

$38 \mathrm{f}$<smiles>O=C1C(Nc2cc(C(F)(F)F)cc(C(F)(F)F)c2)C(=O)C1NC1CCCCC1N1CCCCC1</smiles>

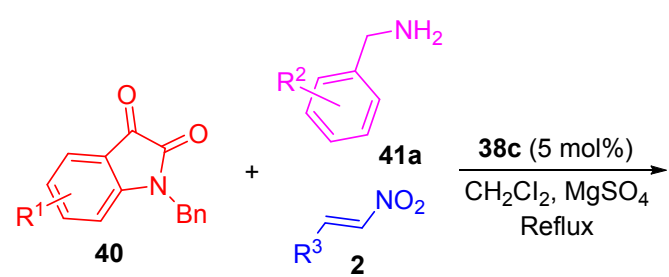<smiles>[R1]c1ccc(C2NC(C(=O)O)(C(=O)N(Cc3ccccc3)c3ccc([R1])cc3)C([2H])C2[N+](=O)[O-])cc1</smiles>

18 samples, $58 \% \sim 77 \%$ yield 5:1 11:1 dr, 77\% 96\% ee

基烯(2)、吲哚(18)和 3-重氮吲哚(35b)的三组分不对称串 联反应, 以 $64 \% \sim 99 \%$ 的产率、 $78 \% \sim 99 \%$ 的 $e e$ 值合成 了一系列手性双吲哚化合物 41. 基于该方法学研究, 得 到的化合物 41a 可经 6 步以 $14.5 \%$ 的总产率实现对生物 碱(一)-folicanthine (42) 的不对称全合成, 发展了构建手 性双吲哚类生物碱的新方法.

多功能团化手性哌啶衍生物是一类重要的有机反 应合成子, 是合成多种重要复杂手性化合物的关键中间 体 ${ }^{[32]}$. 2014 年, Blümel 等 ${ }^{[33]}$ 利用双功能手性方酰胺奎宁胺 38e 作催化剂, 发展了 1,3 -二羰基化合物(30)、 $\beta$ 硝基烯烃(2)和亚胺醛(7)的三组分不对称 Michael/azaHenry/cyclization 串联反应. 反应首先通过 $38 \mathrm{e}$ 的氢键 催化形成中间体 I, 然后进一步合成了一类具有潜在生 物活性的四氢吡啶衍生物 $\mathbf{4 3}$ (Scheme 12). 该方法具有 催化剂用量少 $(0.5 \mathrm{~mol} \%)$ 、条件温和、底物普适性好、 对映异构体选择性( $93 \%$ ～99\% ee) 优异等优点，且将反<smiles>[R]NCC([13CH3])NC1C(=O)C(=O)C1Nc1cc(C(F)(F)F)cc(C(F)(F)F)c1</smiles>

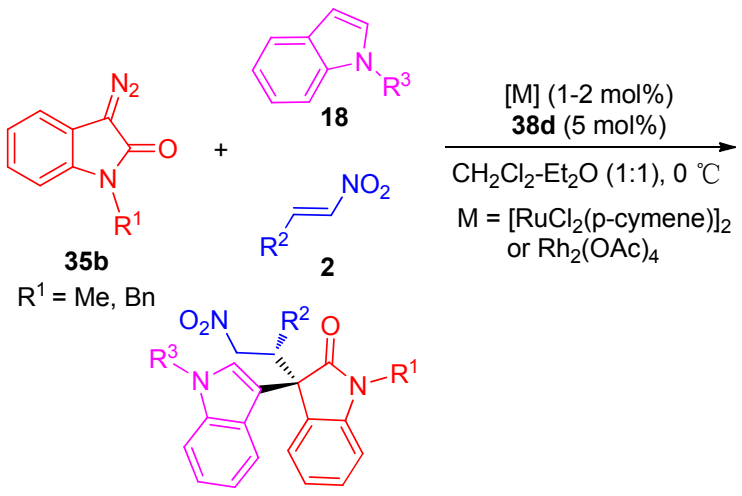

41 (19 samples) $64 \% \sim 99 \%$ yield up to $20: 1 d r, 78 \% \sim 99 \%$ ee
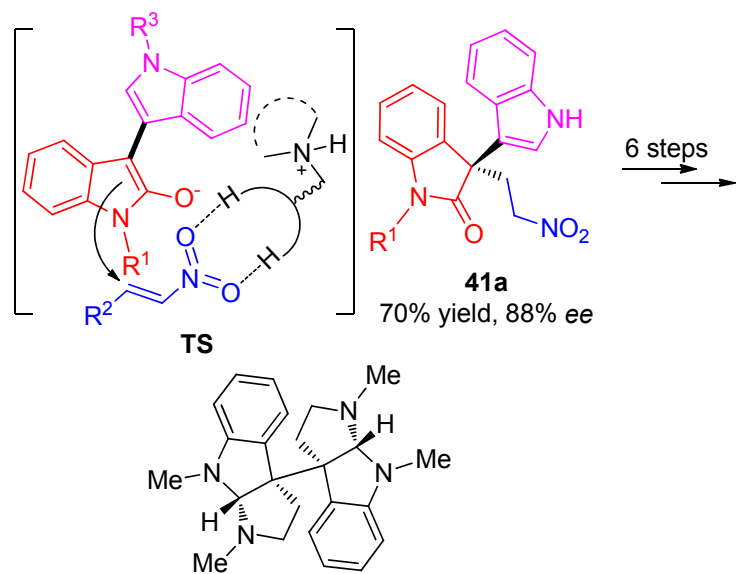

(-)-folicanthine (42)

$14.5 \%$ overall yield

图式 11 基于 $\mathrm{C}-\mathrm{H}$ functionalization/Michael 串联反应合成吲 哚衍生物 41

Scheme 11 Asymmetric synthesis of chiral indole derivatives 41 based on $\mathrm{C}-\mathrm{H}$ functionalization/Michael cascade reaction 
应量增加到 “克” 量级都不影响产物的产率和立体选择 性, 具有潜在的工业应用价值. 不足之处是部分产率和 非对映异构体选择性不够理想.<smiles>[R]C=C[N+](=O)[O-]</smiles>

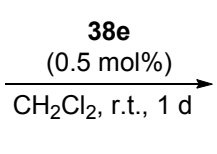

2<smiles>[R]C(=O)C1=C([R])N([R])C([R])[C@H]([R])[C@H]1[N+](=O)[O-]</smiles>

图式 12 有机催化多组分 Michael/aza-Henry/cyclization 串联 反应合成哌啶衍生物 $\mathbf{4 3}$

Scheme 12 Asymmetric synthesis of tetrahydropyridines (43) via organocatalytic multicomponent Michael/aza-Henry/cyclizetion cascade reaction

随后, Blümel 等 $^{[34]}$ 又发展了双功能手性方酰胺一奎 宁胺催化的三组分 “一锅法” 不对称 Michael/Michael/ aldol 串联反应(Eq. 6). 该反应基于方酰胺-奎宁胺 $\mathbf{3 8 b}$ (1 mol\%)对硝基烯(2)和 1,3-二羰基化合物(30b)的双重 氢键作用，再与化合物 44 发生串联反应，合成了一系列 具有潜在生物活性的螺环 1,3-狮满二酮衍生物 45. 该方 法的局限在于底物的普适性不高, 反应只取得了中等的 产率( $23 \% \sim 72 \%)$ 和立体选择性, 部分例子的非对映异 构体选择性 $(d r$ 值) 不够理想.

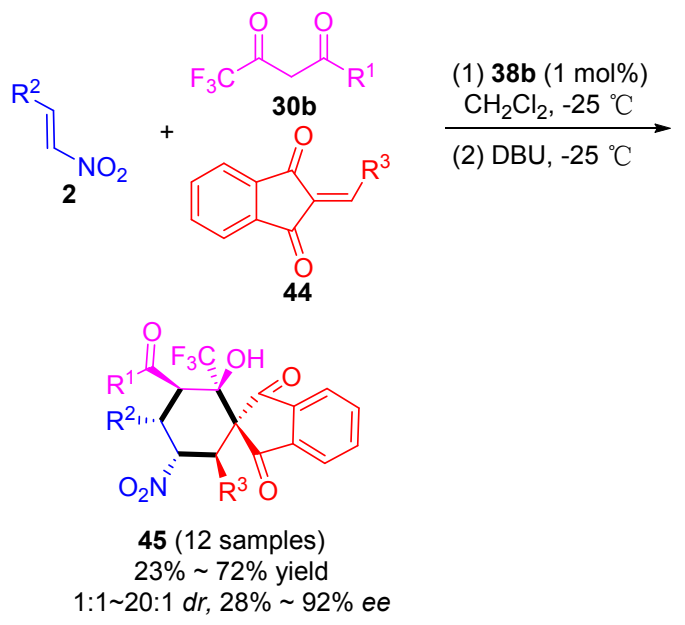

手性四氢吡喃广泛存在于天然产物和药物分子中, 基于有机不对称催化构建手性四氢吡喃骨架的新方法 是有机及药物化学研究的重要课题 ${ }^{[35]}$. Enders 课题组 ${ }^{[36]}$ 于 2014 年报道了双功能方酰胺-奎宁胺 38d 催化的三组
分 “一锅法” 不对称 Michael/Henry/ketalization 串联反 应(Eq. 7). 该方法从 1,3-二羰基化合物 30 与硝基烯烃(2) 出发, 经双功能方酰胺-奎宁胺 38d 催化发生不对称 Michael 加成反应，加成产物 I 进一步与炔醛化合物 46 发生 Henry/ketalization 反应，再经 $p$-TSA 催化，以优异 的对映异构体选择性( $93 \%$ \% $99 \%$ ee ) 获得含有五个手性 中心的四氢吡喃衍生物 47. 该方法的不足在于非对映 异构体选择性 $(d r$ 值)不是很理想.

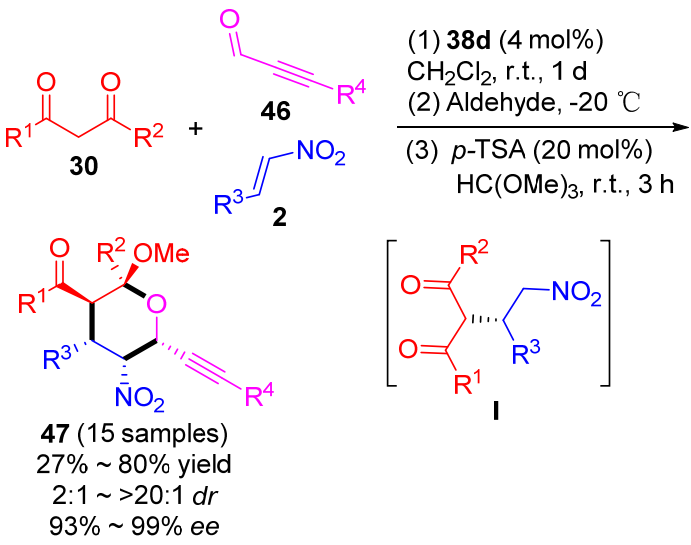

同年, Enders 课题组 ${ }^{[37]}$ 开展了基于催化剂构型诱导 的对映体合成研究，合成了两类构型相反、含有六个连 续立体中心的螺吡唑啉酮的对映体化合物 $48 \mathrm{a}$ 和 $48 \mathrm{~b}$ (Scheme 13). 该方法以 $1 \mathrm{~mol} \%$ 方酰胺-奎宁胺 $(38 d$ 或 38e) 为催化剂, 通过 1,3-二羰基化合物 30a、硝基烯烃(2) 和不饱和吡唑啉酮 49 的三组分不对称 Michael/Michael/ 1,2-addition 串联反应, 取得了优异的立体选择性(10： $1 \sim>25: 1 d r, 97 \% \sim 99 \% e e)$. 该方法具有催化剂用量 少、立体选择性优异、底物普适性强等优点. 此外, 该 方法还可实现产物的 “克” 量级(2.56 g) 反应而不影响其 立体选择性 $(>25 ： 1 d r, 99 \% e e)$. 当以 2-氧代环戊烷羧 酸甲酯(30aa)作为 1,3 -二羰基化合物参与该多组分串联 反应时，能以良好的立体选择性( $>25 ： 1 d r, 95 \% e e)$ 构 建三个手性季碳中心, 但其位阻因素可能影响了产物的 产率(16\%).

Enders 研究组 ${ }^{[38]}$ 进一步探究了催化剂构型诱导的 不对称合成研究, 并发展了双功能方酰胺-奎宁胺与非 手性碱三氮杂双环癸烯 (TBD) 协同催化的多组分 Michael/Michael/1,2-addition 串联反应的新方法(Eq. 8). 该方法以 $1 \mathrm{~mol} \%$ 的手性方酰胺-奎宁胺 $(\mathbf{3 8 d}$ 或 $\mathbf{3 8 e})$ 为催 化剂，发展了硝基烯烃(2)、 $\beta$-羰基羧酸酯(30a)和 $\alpha, \alpha$-二 氰基乙烯衍生物 $(\mathbf{5 0})$ 的三组分不对称串联反应. 反应最 终以 $68 \% \sim 86 \%$ 的产率、优异的非对映异构体选择性 $(>$ $30 ： 1 d r)$ 和对映异构体选择性(96\% 99\% ee) 获得了含 有五个手性中心的多功能团化手性环己烷衍生物 51. 


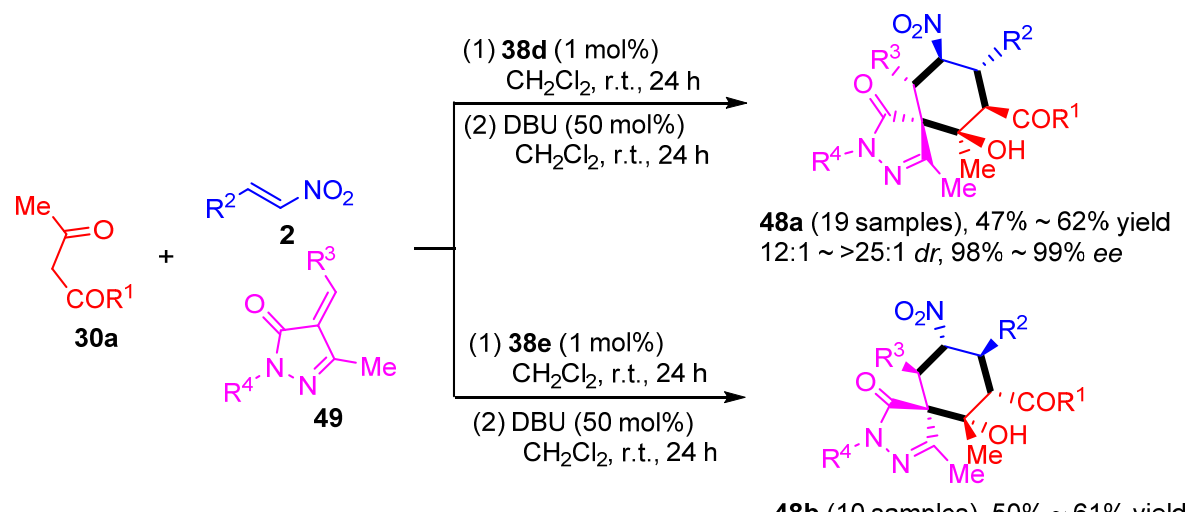

48b (10 samples), $50 \% \sim 61 \%$ yield $10: 1 \sim>25: 1 d r, 97 \% \sim 99 \%$ ee

图式 13 有机催化三组分 Michael/Michael/1,2-addition 不对称串联反应合成手性螺吡唑啉酮衍生物 48a 和 48b

Scheme 13 Asymmetric organocatalytic three-component Michael/Michael/1,2-addition cascade reaction to synthesize chiral spiropyrazolones derivatives $\mathbf{4 8 a}$ and $\mathbf{4 8 b}$<smiles>[R]C=[N+]([O-])[N+](=O)CCC(C)=O</smiles>

(1) $38 \mathrm{~d}$ or $38 \mathrm{e}(1 \mathrm{~mol} \%)$

2

$$
\begin{aligned}
& \text { or ent-51 } \\
& \text { 68\% } \sim 86 \% \text { yield } \\
& >30: 1 d r, 96 \% \sim 99 \% \text { ee }
\end{aligned}
$$

随后，另一例基于三组分不对称 1,4-/1,6-/1,2-addition 串联反应合成全取代手性环已烷衍生物 52 的方法 也被 Enders 研究组报道 ${ }^{[39]}$ (Eq. 9). 该方法通过双功能 方酰胺-奎宁胺 $(\mathbf{3 8 d}$ 或 38e) 与非手性碱 DBU 协同催化, 建立了 $\beta$-硝基烯烃(2)、 $\beta$-二羰基化合物(30a) 和 4-硝基-5烯基异噁唑(53)的三组分串联反应. 该反应以微量的催 化剂用量 $(1 \mathrm{~mol} \%)$ 、优异的对映异构体选择性 $(91 \%$ 99\% ee) 得到了具有六个连续立体中心的异噁唑取代环 己烷衍生物 52. 研究发现, 当把反应量放大到 $8 \mathrm{mmol}$ 时, 只需 $0.5 \mathrm{~mol} \%$ 的催化剂用量, 在温和的条件下就能 以 $57 \%$ 的产率突出的立体选择性( $>20: 1 d r, 98 \% e e$ ) 获 得目标化合物, 具有潜在的开发价值.

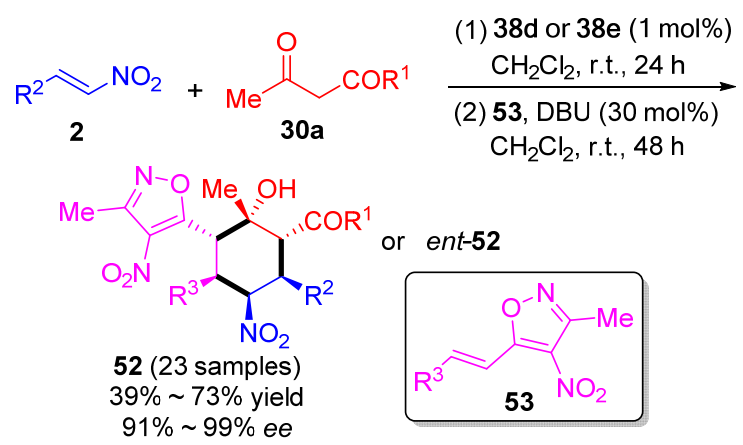

2015 年, 孙兴文课题组 ${ }^{[40]}$ 建立了基于双功能方酰 胺-奎宁胺 38d 催化的三组分不对称 Michael/Michael/ aldol 串联反应的合成方法, 并成功应用于多取代 3-环 己烷螺环氧化吲哚衍生物 54 的合成(Eq. 10). 该方法以 硝基烯(2)、1,3-二羰基化合物(30)和 $N$-Boc 氧化吲哚烯 衍生物(55)为原料, $5 \mathrm{~mol} \%$ 的 $\mathbf{3 8 d}$ 为催化剂, 在温和的 条件下，以优异的立体选择性 $(>20: 1 d r,>99 \% e e)$ 合 成了一系列全取代的手性螺环吲哚衍生物 54, 为手性 螺环吲哚在医药领域的进一步研究提供了丰富的化合 物篎选库.

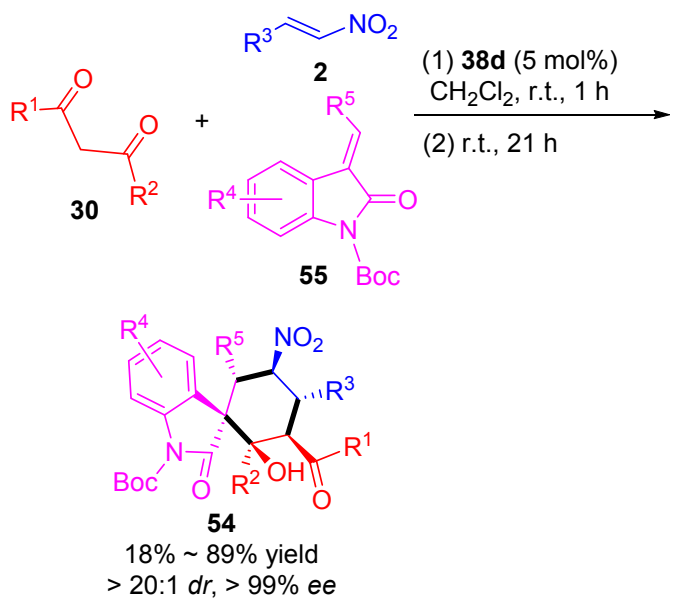

随后，该课题组进一步研究发现，当把上例反应中 的底物吲哚烯 55 换为 $\alpha, \beta$-不饱和醛 13 时, 在方酰胺奎宁胺 38d $(0.5 \mathrm{~mol} \%)$ 与非手性吡咯烷 $(10 \mathrm{~mol} \%)$ 的双催 化作用下，通过 Michael/Michael/aldol 串联反应，能以 良好的立体选择性 $(>20: 1 \mathrm{dr},>99 \% \mathrm{ee})$ 获得一类含 有六个连续手性中心的全取代环己烷衍生物 $\mathbf{5 6}^{[41]}$ (Eq. 11). 在反应中, 方酰胺-奎宁胺 38d 通过 $\mathrm{NH}$ 基团与硝基 形成氢键作用产生立体效应，奎宁结构的仲胺则通过氢 
键对乙酰丙酮 (30c) 的羰基进行活化, 发生不对称 Michael 加成(如 TS 所示). 得到的化合物 56 含有醛基、 硝基、乙酰基、羟基等反应活性基团，是构建多种复杂 手性分子的重要中间体.

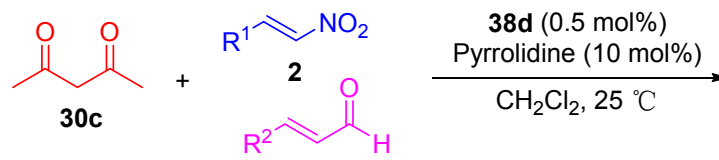

13
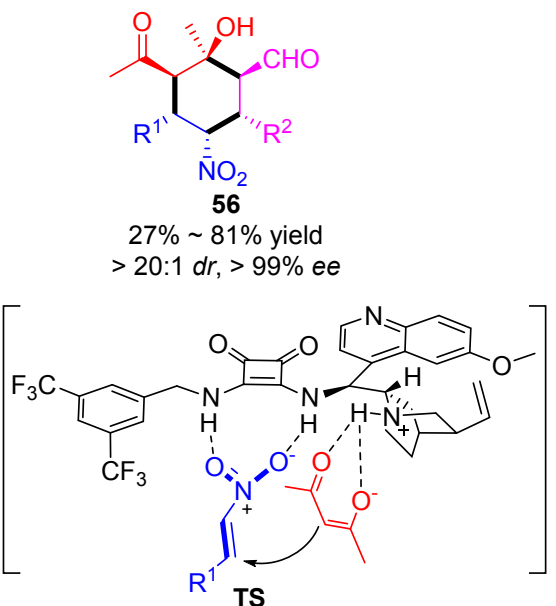

色满骨架普遍存在于 $(+)$-catechin、( - )-siccanin 等 天然产物成分中 ${ }^{[42]}$, 许鹏飞课题组 ${ }^{[43]} 2016$ 年建立了手 性芳酰胺-奎宁胺 38d 催化的三组分不对称 $[3+3] \mathrm{annu}-$ lation/nitro-Michael 串联反应，合成了一类新型 3-硝基 色满衍生物 57 (Eq. 12). 该方法从苯酚衍生物 58、2-硝 基-1,3-烯炔(2e) 与芳基乙烯酮(59)出发, 以中等的产率 $(20 \% \sim 90 \%)$ 、良好的立体选择性(>25：1 $d r, 94 \%$ $99 \%$ ee) 合成了 21 个 3 -硝基色满衍生物 57. 该反应具有 良好的底物普适性，实验表明当将反应投量扩大到“克” 量级时, 反应依然能够顺利进行, 且不影响产物的产率 和立体选择性，具有潜在的工业开发应用价值.<smiles>[R]C#C/C(=C/[R])[N+](=O)[O-]</smiles>

(1) $38 \mathrm{~d}$ (10 $\mathrm{mol} \%)$

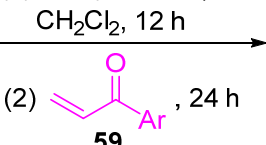<smiles>[R]C=C1Oc2cc[R1]cc2C([R10])C1(O)[C@@H](O)CC(=O)[Al]</smiles>

21 samples, $20 \% \sim 90 \%$ yield

$>25: 1 d r, 94 \% \sim 99 \%$ ee

3-氨基-3-烷基氧化吲哚是一类重要的 3,3'-螺环 氧化吲哚衍生物, 是合成多种复杂生物碱的关键中 间体 ${ }^{[44]}$. 韩志勇课题组 ${ }^{[45]}$ 于 2016 年报道了有机金属
催化的 3-重氮吲哚(35c)、仲胺(41b)和硝基乙烯(2f) 的三组分卡宾 $\mathrm{N}-\mathrm{H}$ 功能化/Michael 不对称串联反应 (Scheme 14). 反应以 $2 \mathrm{~mol} \% \mathrm{Ru}(\mathrm{II})$ 与 $10 \mathrm{~mol} \%$ 的手性 双功能方酰胺-奎宁胺 38d 为催化剂, 构建了一类高 光学纯 $(83 \% \sim 95 \% e e)$ 的 3-氨基-3-烷基氧化吲哚衍生 物 60. 以化合物 60a 为关键中间体，最终以 $25 \%$ 的总 产率实现了生物碱(一)-psychotrimine 的全合成，从而 实现了不对称串联反应在天然产物全合成中的应用.
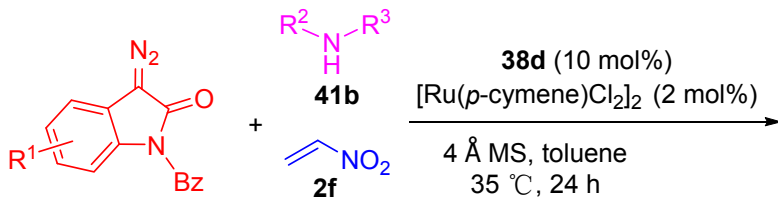

$35 c$

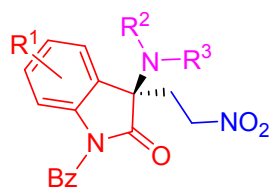

60 (15 samples)

$54 \% \sim 72 \%$ yield $83 \% \sim 95 \%$ ee

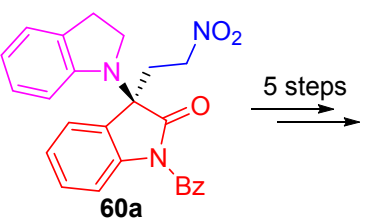

$72 \%$ yield, $92 \%$ ee

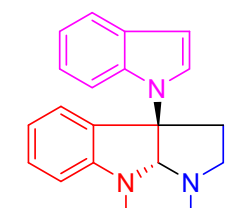

$\mathrm{Boc} \mathrm{Me}$

61

$$
\stackrel{8 \text { steps }}{\longrightarrow}(-) \text {-psychotrimine }
$$

图式 14 有机催化三组分卡宾 $\mathrm{N}-\mathrm{H}$ 功能化/Michael 不对称 串联反应

Scheme 14 Enantioselective organocatalytic three-component carbenoid-associated $\mathrm{N}-\mathrm{H}$ functionalization/Michael-addition cascade reaction

Urruzuno 等 ${ }^{[46]}$ 于 2017 年报道了双功能手性方酰 胺-奎宁胺 38 催化的三组分不对称 $[2+2+2]$ 串联反应, 取得了优异的催化效果(Scheme 15). 在催化剂 38c 或 38f 的催化作用下, $\beta$-四氢荎酮(62)和一分子硝基烯(2)发 生不对称 Michael 加成得到中间体 $\gamma$-硝基酮(63). 经催化 剂 38c 的氢键活化, $\gamma$-硝基酮(63) 可与另一分子硝基烯(2) 发生串联反应，以 $99 \%$ 的对映异构体选择性得到具有六 个连续手性中心的三环化合物 64. 此外, $\gamma$-硝基酮(63)也 可与丙烯醛(13a)发生环化反应得到螺环化合物 65.

\section{2 双功能方酰胺-手性二胺催化的多组分不对称串 联反应}

吡唑啉酮衍生物具有退热止痛、抗 HIV、抗菌、抗 病毒等多种药用活性 ${ }^{[47]}$, 杜大明课题组 ${ }^{[48]}$ 于 2016 年开 展了手性方酰胺-反式环己二胺 $\mathbf{3 8 g}$ 和二苯基 $L$-脯胺醇 


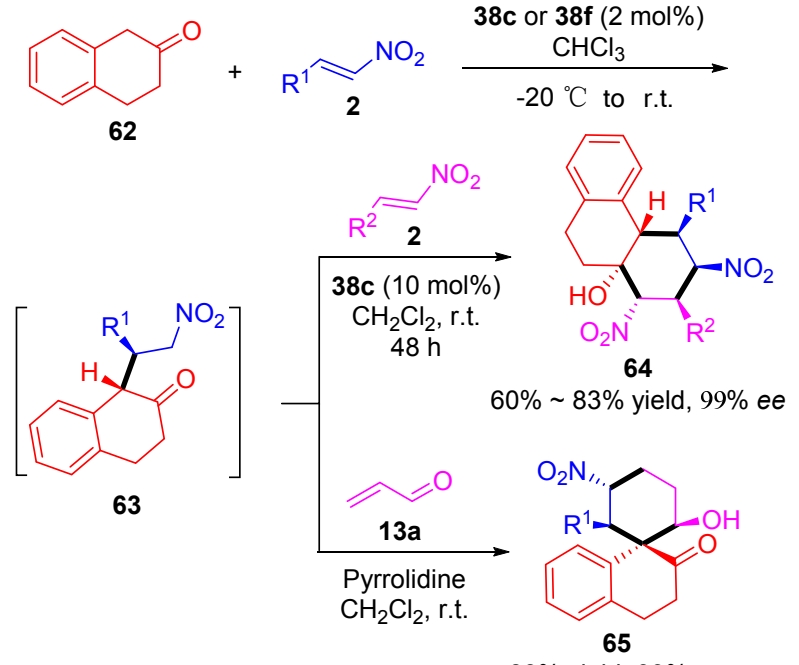

$83 \%$ yield, $99 \%$ ee

图式 15 有机催化三组分不对称 $[2+2+2]$ 串联反应

Scheme 15 Asymmetric organocatalytic three-component $[2+$ $2+2$ ] cascade reaction

硅 12d 协同催化的不对称 Michael/Michael/aldol 串联反 应, 合成了含有四个连续手性中心的吡唑啉酮衍生物 66 (Eq. 13). 反应基于硝基烯烃(2)、 $\alpha, \beta$-不饱和醛(13)和 吡唑啉酮类化合物(67)的三组分不对称串联反应, 以中 等产率(30\% 78\%)获得了优异的立体选择性(60：40～ $25: 1 d r, \geq 99 \% e e)$. 该反应首先在催化剂 $\mathbf{3 8 g}$ 的叔胺催 化作用下发生化合物 67 的去质子化, 同时方酰胺的两 个 $\mathrm{NH}$ 基团与硝基烯(2)发生双氢键活化得到过渡态 TS, 去质子化的 67 与硝基烯发生不对称 Michael 加成, 再与 化合物 13 发生分子间不对称 Michael 加成及分子内 aldol 反应得到化合物 66.<smiles>[R]C=CC=C[N+](=O)[O-]</smiles><smiles>FC(F)(F)c1ccc(C(F)(F)F)c(C(F)(F)F)c1</smiles>

噻唑酮衍生物普遍存在于天然产物分子和药物活 性成分中, 具有多种生物活性 ${ }^{[49]}$. 周正洪研究组 ${ }^{[50]}$ 于 2018 年建立了双功能手性方酰胺-叔胺催化的硝基烯 (2)、丙烯醛(13a)和噻唑-4-酮(67)的三组分 Michael/ Michael/Henry 不对称串联反应，合成了两类构型不同 的双环手性螺环噻唑酮衍生物 68 和 69 (Eq. 14). 该方法

通过方酰胺一叔胺 $38 \mathrm{~h}$ 对硝基烯的氢键活化，以良好的 立体选择性构建了两类具有潜在生物活性的手性噻唑 酮对映体 68 和 69. 该方法能将反应物增加至克量级并 取得良好的产率和对映异构体选择性( $>99 \% \mathrm{ee})$, 为新 型噻唑衍生物的合成及应用研究奠定了基础.
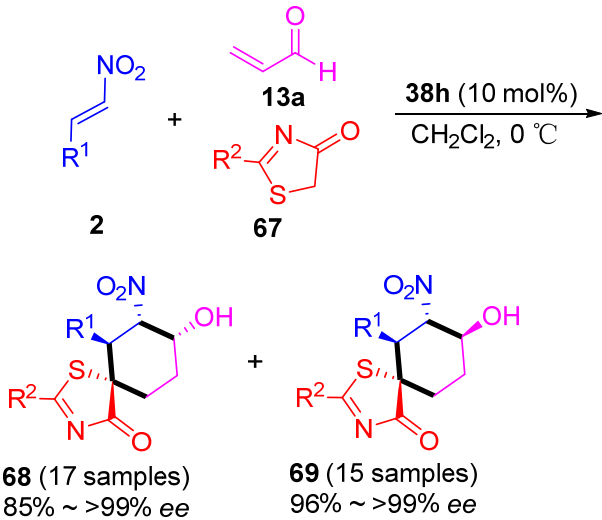

\section{3 其它双功能催化剂催化的多组分不对称串联}

\section{反应}

除双功能硫脲一胺催化剂、方酰胺-胺催化剂外, 手 性 BINOL-磷酸(70)和功能化多孔有机微球化合物(71 和 72)、胺-硫代磷酰胺(73)和手性胍-胺(74)也可作为双功 能催化剂, 在氢键活化的硝基烯参与的多组分不对称串 联反应中有一定的应用.

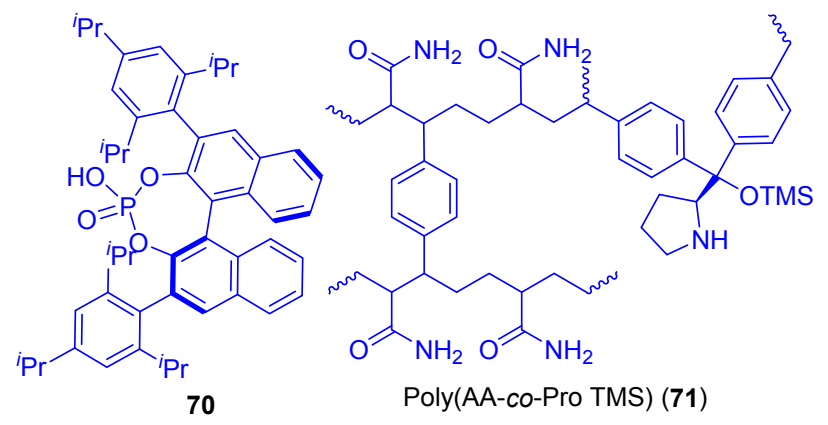

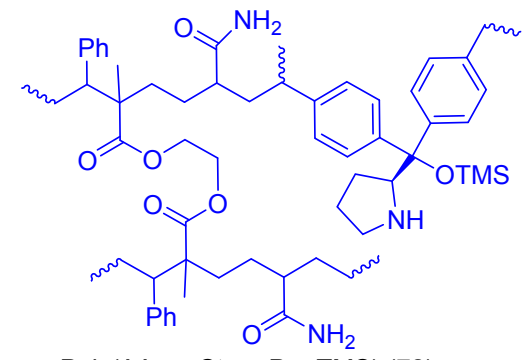

Poly(AA-co-St-co-Pro TMS) (72)<smiles>NC(c1ccccc1)C(NP(=S)(c1ccccc1)c1ccccc1)c1ccccc1</smiles>

73

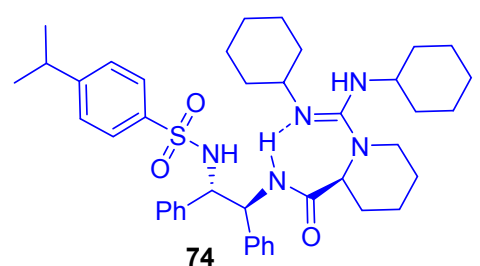




\section{1 双功能手性 BINOL-磷酸催化的多组分不对称串 联反应}

手性磷酸是一类重要的双功能有机催化剂, 具有提 供质子和接受质子的双功能作用，可以同时活化两个反 应底物 ${ }^{[51]}$. Terada 和 Akiyama 等 ${ }^{[52]}$ 于 2004 年分别报道 了手性联二萗酚(BINOL)衍生的手性磷酸催化剂在不对 称催化反应中的应用. 由于手性 BINOL-磷酸在多组分 不对称串联反应中表现出高效、高立体选择性的催化活 性，因而受到人们的普遍关注 ${ }^{[53]}$.

2015 年, 王保民研究组 ${ }^{[54]}$ 建立了手性 BINOL-磷酸 (70)氢键催化的三组分不对称 1,3-偶极环加成反应合成 新型手性螺环氧化吲哚 75 的新方法, 并合成了 26 个目 标化合物. 该方法不仅具有良好的底物普适性, 且当反 应放大到 “克” 量级时仍可取得优异的产率(99\%)、优 异的非对映异构体选择性(12:1 $d r)$ 和对映异构体选择 性(95\% ee), 手性螺环氧化吲哚 75a 可进一步转变为 77 及 78, 在药物合成领域中具有重要的应用价值(Scheme 16).
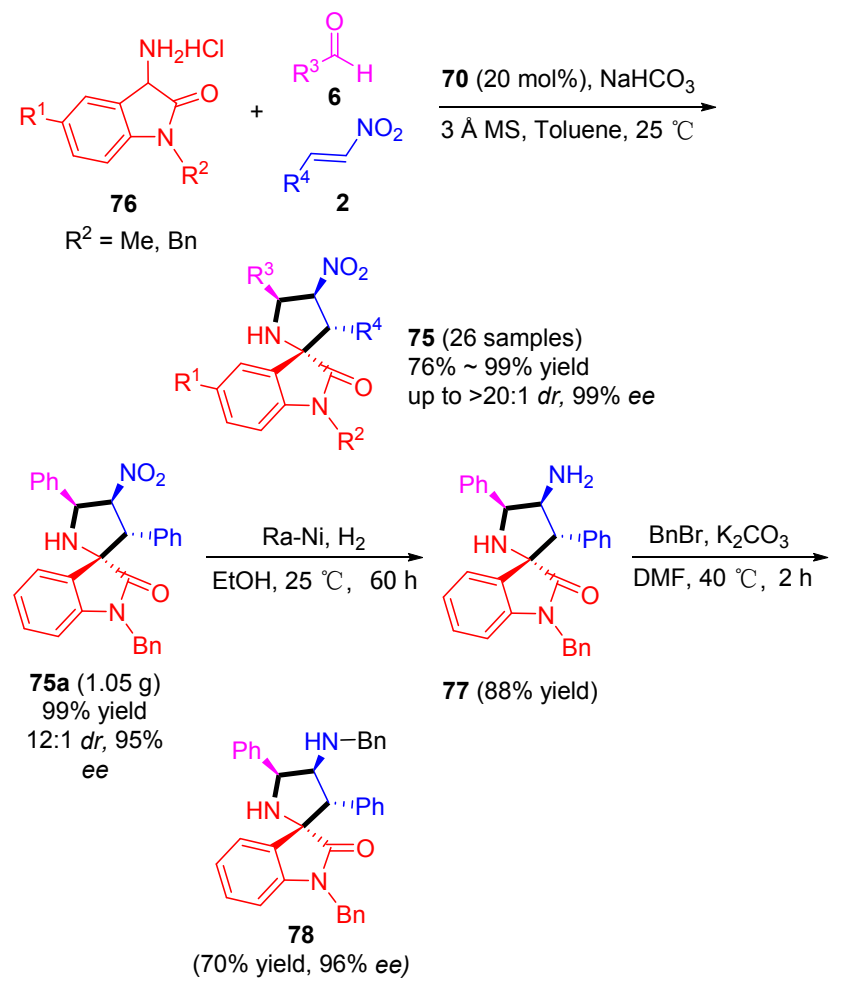

图式 16 手性 BINOL-磷酸 70 催化的三组分不对称 1,3-偶极 环加成反应合成手性螺环氧化吲哚衍生物 75

Scheme 16 Enantioselective synthesis of chiral spirooxindole derivatives 75 via chiral BINOL-phosphoric acid (70) catalyzed three-component 1,3-dipolar cycloaddition reaction

随后, 王保民研究组 ${ }^{[55]}$ 深入开展了基于双功能氢 键催化并用于合成手性螺环吲哚衍生物的方法学研究, 于 2018 年开发了 3-硝基- $2 H$-色烯(2g)、醛(6)和吲哚甲亚 胺内鎓盐(76a)的三组分不对称 $[3+2]$ 环加成反应，合成 了一系列新型 3,3'-吡咯螺环氧化吲哚衍生物 79 (Eq. 15). 该方法利用手性 BINOL-磷酸 70 为催化剂、 $\mathrm{NaHCO}_{3}$ 为 碱，在室温条件下，以优异的产率(82\% 99\%)和立体 选择性(up to $>20 ： 1 d r, 96 \% e e$ )构建了四个手性中心 (含两个手性季碳中心). 在反应中, 催化剂 70 通过羟基 与硝基基团形成氢键以提高化合物 $\mathbf{2 g}$ 的反应活性，同 时磷酰基也通过氢键活化甲亚胺内鎓盐中间体(如 TS 所 示)，在 $[3+2]$ 环加成反应中起到手性控制的作用. 该方 法放大到 “克” 量级时，仍可取得 $78 \%$ 的产率和良好的 选择性 $(76 \% e e,>20: 1 d r)$, 具有潜在的工业开发利用 价值.<smiles>[R]c1ccc2c(c1)C(N)C(=O)N2Cc1ccccc1</smiles><smiles>[R]C(=O)C[N+](=O)[O-]</smiles>
$70\left(20 \mathrm{~mol}^{\%}\right)$
$\underset{3 \mathrm{NaHCO}}{\mathrm{A} \mathrm{MS}, \text { Toluene }}$ $76 a$ $2 \mathrm{~g}$

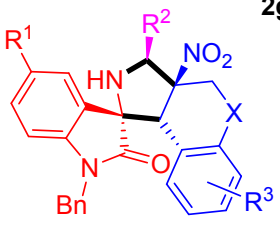

79 (21 samples) $82 \% \sim 99 \%$ yield up to $>20: 1 d r, 96 \%$ ee

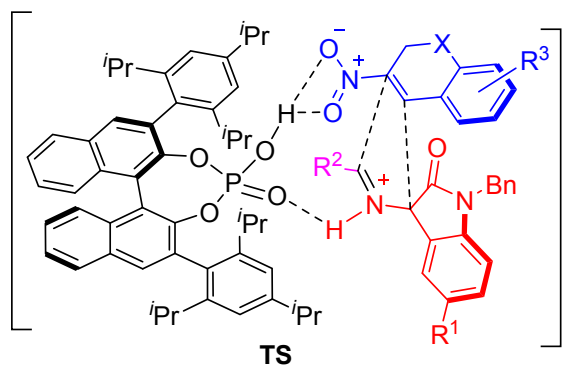

\section{2 功能化多孔有机微球催化的多组分不对称串联反 应}

多功能化有机微球催化的多组分不对称串联反应 具有高效、环境友好、催化剂可循环再利用等优点 ${ }^{[56]}$. 西南大学马学兵研究组 ${ }^{[57]}$ 于 2017 年报道了 JørgensenHayashi 功能化的多孔有机微球 71 和 72 的合成、性能 表征及在脂肪醛 $(6)$ 、硝基烯 $(2)$ 和肉桂醛 $(13 b)$ 的三组分 不对称 Michael/Michael/aldol 串联反应中的催化应用 (Eq. 16). 基于多功能化有机微球与底物的多重氢键活 化，反应以良好的产率( $31 \% \sim 61 \%$ )、优异的立体选择性 (80：20 93：7 dr, >99\% ee)合成了一系列多取代手 性环己烯醛衍生物 80. 研究发现该微球催化剂可反复 使用多次后仍具有良好的催化性能. 

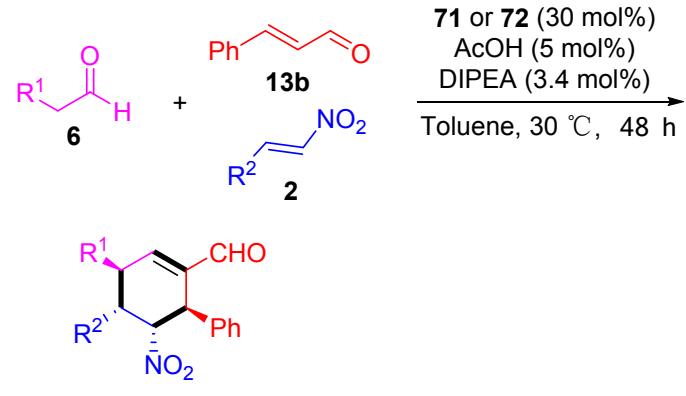

80

$31 \% \sim 61 \%$ yield

$80: 20 \sim 93: 7 d r,>99 \%$ ee

\section{3 双功能胺-硫代磷酰胺催化的多组分不对称串联} 反应

胺一硫代磷酰胺是一类重要的双功能手性催化剂, 其结构中的手性胺可以通过氢键作用、烯胺活化等方式 对多个反应底物进行选择性活化, 实现对产物的立体选 择性控制 ${ }^{[58]}$. 周正洪研究组 ${ }^{[59]}$ 于 2016 年开发了新型胺硫代磷酰胺 73 催化的三组分不对称 Michael/Michael/ aldol 串联反应, 合成了 22 个具有潜在生物活性的螺环 吡唑酮衍生物 82 (Eq. 17). 该反应在催化剂 73 的烯胺活 化和氢键作用下(如 TS 所示), 首先发生丙酮 (27a)和硝 基烯(2)的不对称 Michael 加成, 得到的中间体 I 再与化 合物 81 发生不对称 Michael/aldol 反应, 取得了突出的立

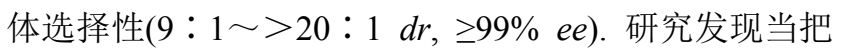
反应物的用量增大到“克”量级时仍能取得理想的催化 效果, 并以 $65 \%$ 的产率和优异的立体选择性( $>20: 1$ $d r,>99 \%$ ee) 合成了 $6.55 \mathrm{~g}$ 的螺环吡唑酮衍生物 82a.<smiles>[R]C=C1C(=O)N(c2ccccc2)N=C1[R]</smiles>

(1) 73 (10 mol\%), Toluene r.t., $16 \sim 59 \mathrm{~h}$

(2) $\mathrm{DBU}$ (1 equiv.) $\mathrm{CH}_{2} \mathrm{Cl}_{2}$, r.t.<smiles>[R]C(CC(=O)CC)C[N+](=O)[O-]</smiles><smiles>[R]C1=CN(c2ccccc2)C(=O)C12C([R])C(O)C([R])[C@@H]([N+](=O)[O-])C2[R]</smiles>

82 (22 samples) $42 \% \sim 67 \%$ yield 9:1 >20:1 dr, $\geq 99 \%$ ee

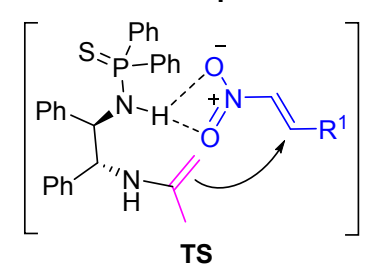

\section{4 双功能手性胍一胺催化的多组分不对称串联反应}

双功能手性胍一胺催化剂具有多个手性胺结构, 在 有机催化反应中可通过氢键作用对多个反应底物进行 选择性活化，实现反应的立体选择性控制 ${ }^{[60]} .2017$ 年, 冯小明研究组 ${ }^{[61]}$ 建立了新型双功能手性胍-胺 74 催化
的 $\alpha$-酮酸酯 $(83)$ 和两分子硝基烯 (2) 的三组分不对称 Michael/Michael/Henry 串联反应(Eq. 18). 以 56\% 99\% 的产率、良好的立体选择性( $>20: 1 d r, 83 \% \sim 95 \% e e$ )构 建了含有六个相邻立体中心的全取代环己烷衍生物 84. 在反应中，催化剂 74 通过氢键和 Brønsted 碱的协同催 化对双 Michael/Henry 串联反应进行活化(如 TS 所示), 其合成的手性环已烷衍生物 84 可进一步进行官能团转 化，作为重要反应中间体用于多种复杂手性化合物的合 成.
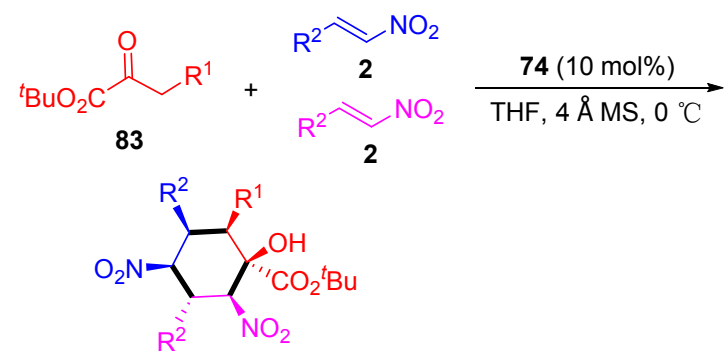

$$
\begin{aligned}
& 84 \text { (23 samples) } \\
& 56 \% \sim 99 \% \text { yield }
\end{aligned}
$$

$>20: 1 d r, 83 \% \sim 95 \%$ ee

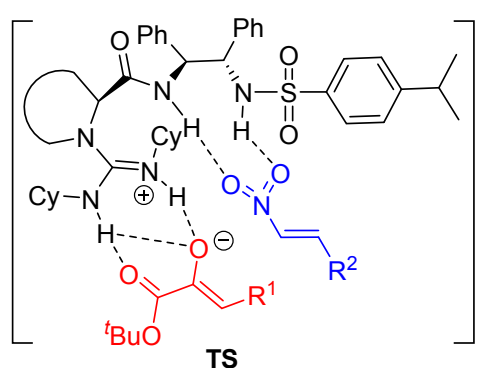

\section{4 总结与展望}

本文系统地综述了基于双功能硫脲-胺、方酰胺-胺 和其它双功能催化剂对硝基烯进行氢键活化, 并作为主 要合成子参与的多组分不对称串联合成研究进展, 详细 地介绍了反应类型、反应机理、反应特点及应用等. 这 些研究实例表明, 双功能手性催化剂通过氢键活化的多 组分不对称串联反应具有环境友好、操作简洁、立体选 择性好、原子经济性高、不需中间体的分离纯化等优点， 是构建类型多样、结构复杂的手性化合物的最有效合成 方法之一.

尽管该研究领域已取得较大进展, 但仍存在进一步 研究和发展的空间: (1)目前已报道的双功能手性催化剂 存在类型少、使用局限等问题，更多高效的新型双功能 手性催化剂有待开发; (2)基于双功能手性催化剂催化的 多组分不对称串联反应类型有待丰富，基于催化机理开 发多组分串联新反应仍是有机合成的重要研究方向; (3) 该类反应在新药开发和复杂天然产物不对称全合成中 的应用还存在较大空间. 随着化学工作者对有机反应研 
究的深入, 我们相信更多新型的双功能催化剂、更高效 的多组分不对称串联合成新方法将会被研究报道, 并将 被成功应用于新分子合成、新药研发、新材料发现及其 它相关领域.

\section{References}

[1] For some typical references in this area, see:

(a) Volla, C. M. R.; Atodiresei, I.; Rueping, M. Chem. Rev. 2013, 114, 2390 .

(b) Chauhan, P.; Mahajan, S.; Kaya, U.; Hack, D.; Enders, D. Adv. Synth. Catal. 2015, 357, 253.

(c) Yan, L. J., Wang, Y. C. ChemistrySelect 2016, 1, 6948.

(d) Cao, Y.; Jiang, X.; Liu, L.; Shen, F.; Zhang, F.; Wang, R. Angew. Chem., Int. Ed. 2011, 50, 9124.

(e) Li, X.; Guo, L.; Peng, C.; Han, B. Chem. Rec. 2019, 19, 394.

(f) Vinogradov, M. G.; Turova, O. V.; Zlotin, S. G. Org. Biomol. Chem. 2019, 17, 3670.

(g) Wu, X.; Li, M. L.; Gong, L. Z. Acta Chim. Sinica 2013, 71, 1091 (in Chinese).

(吴祥, 李明丽, 龚流柱, 化学学报, 2013, 71, 1091.)

(h) Han, Z. Y.; Gong, L. Z. Prog. Chem. 2018, 30, 505 (in Chinese). (韩志勇, 龚流柱, 化学进展, 2018, 30, 505.)

[2] (a) Shibasaki, M.; Matsunaga, S. Chem. Soc. Rev. 2006, 35, 269.

(b) Georgiou, I.; Ilyashenko, G.; Whiting, A. Acc. Chem. Res. 2009, 42,756 .

(c) Asano, K.; Matsubara, S. J. Am. Chem. Soc. 2011, 133, 16711.

(d) Xiao, Y. C.; Chen, F. E. ChemCatChem 2019, 11, 2018.

(e) Yu, X.; Wang, W. Chem.-Asian J. 2008, 3, 516.

(f) Held, F. E.; Tsogoeva, S. B. Catal. Sci. Technol. 2016, 6, 645.

(g) Du, L.; Peng, C.; Liao, J. Acta Chim. Sinica 2013, 71, 1239 (in Chinese).

(杜乐, 曹鹏, 廖建, 化学学报, 2013, 71, 1239.)

[3] Hiemstra, H.; Wynberg, H. J. Am. Chem. Soc. 1981, 103, 417.

[4] Okino, T.; Hoashi, Y.; Takemoto, Y. J. Am. Chem. Soc. 2003, 125, 12672.

[5] For some typical references in this area, see:

(a) Zhan, G.; Du, W.; Chen, Y. C. Chem. Soc. Rev. 2017, 46, 1675.

(b) Okino, T.; Hoashi, Y.; Furukawa, T.; Xu, X.; Takemoto, Y. J. Am. Chem. Soc. 2005, 127, 119.

(c) Liu, J. Y.; Zhao, J.; Zhang, J. L.; Xu, P. F. Org. Lett. 2017, 19, 1846.

(d) Shirakawa, S.; Koga, K.; Tokuda, T.; Yamamoto, K.; Maruoka, K. Angew. Chem., Int. Ed. 2014, 53, 6220.

(e) Chen, P.; Bao, X.; Zhang, L. F.; Ding, M.; Han, X. J.; Li, J.; Zhang, B. B.; Tu, Y. Q.; Fan, C. A. Angew. Chem., Int. Ed. 2011, 50,8161 .

(f) Miyamura, H.; Choo, G. C.; Yasukawa, T.; Yoo, W. J.; Kobayashi, S. Chem. Commun. 2013, 49, 9917.

[6] (a) Barrett, A. G.; Graboski, G. G. Chem. Rev. 1986, 86, 751.

(b) Zimmer, R.; Reissig, H. U. Chem. Soc. Rev. 2014, 43, 2888.

(c) Feng, J.; Li, X. J. Org. Chem. 2017, 82, 7317.

(d) Gao, W.; Lv, H.; Zhang, T.; Yang, Y.; Chung, L. W.; Wu, Y. D.; Zhang, X. Chem. Sci. 2017, 8, 6419.

(e) Zhang, W. S.; Xu, W. J.; Zhang, F.; Li, Y. Chin. J. Org. Chem. 2019, 39, 1277 (in Chinese)

(张文生, 许文静，张斐，李炎，有机化学， 2019，39，1277.)

(f) Yan, L. J.; Xu, H.; Wang, Y.; Dong, J. W.; Wang, Y. C. Chin. J. Org. Chem. 2020, 40, 284 (in Chinese).

(严丽君, 徐菡, 王艳, 董建伟, 王永超, 有机化学, 2020, 40, 284.)

[7] Sigman, M. S.; Jacobsen, E. N. J. Am. Chem. Soc. 1998, 120, 4901.

[8] (a) Pei, Z.; Li, X.; von Geldern, T. W.; Longenecker, K.; Pireh, D.; Stewart, K. D.; Backes, B. J.; Lai, C.; Lubben, T. H.; Ballaron, S. J.; Beno, D. W. A.; Kempf-Grote, A. J.; Sham, H. L.; Trevillyan, J. M. J. Med. Chem. 2007, 50, 1983. (b) Nara, S.; Tanaka, R.; Eishima, J.; Hara, M.; Takahashi, Y.; Otaki, S.; Foglesong, R. J.; Hughes, P. F.; Turkington, S.; Kanada, Y. J. Med. Chem. 2003, 46, 2467.

[9] Imashiro, R.; Uehara, H.; Barbas III, C. F. Org. Lett. 2010, 12, 5250 .

[10] Uehara, H.; Imashiro, R.; Hernández-Torres, G.; Barbas III, C. F. Proc. Natl. Acad. Sci. U. S. A. 2010, 107, 20672.

[11] Basle, O.; Raimondi, W.; Duque, M. D. M. S.; Bonne, D.; Constantieux, T.; Rodriguez, J. Org. Lett. 2010, 12, 5246.

[12] Wang, Y.; Han, R. G.; Zhao, Y. L.; Yang, S.; Xu, P. F.; Dixon, D. J. Angew. Chem., Int. Ed. 2009, 48, 9834.

[13] Mao, Z.; Jia, Y.; Xu, Z.; Wang, R. Adv. Synth. Catal. 2012, 354, 1401.

[14] For recent reviews, see:

(a) Robinson, D. E. J. E.; Bull, S. D. Tetrahedron: Asymmetry 2003 14, 1407.

(b) Keith, J. M.; Larrow, J. F.; Jacobsen, E. N. Adv. Synth. Catal. 2001, 343, 5 .

(c) Reetz, M. T. Angew. Chem., Int. Ed. 2001, 40, 284.

(d) Vedejs, E.; Jure, M. Angew. Chem., Int. Ed. 2005, 44, 3974.

[15] Roy, S.; Chen, K. Org. Lett. 2012, 14, 2496.

[16] Zhou, B.; Yang, Y.; Shi, J.; Luo, Z.; Li, Y. J. Org. Chem. 2013, 78, 2897.

[17] For some typical references in this area, see:

(a) Pearson, W. H.; Hines, J. V. J. Org. Chem. 1989, 54, 4235.

(b) Kingston, D. G.; Samranayake, G.; Ivey, C. A. J. Nat. Prod. 1990, 3, 1 .

(c) Slade, J.; Stanton, J. L.; Ben-David, D.; Mazzenga, G. C. $J$. Med. Chem. 1985, 28, 1517.

(d) Le, V.; Inai, M.; Williams, R.; Kan, T. Nat. Prod. Rep. 2015, 32, 328 .

(e) Jiang, Y.; Chen, X.; Zheng, Y.; Xue, Z.; Shu, C.; Yuan, W.; Zhang, X. Angew. Chem., Int. Ed. 2011, 50, 7304.

(f) Wang, Q.; Huang, W.; Yuan, H.; Cai, Q.; Chen, L.; Lv, H.; Zhang, X. J. Am. Chem. Soc. 2014, 136, 16120.

[18] Hou, W.; Wei, Q.; Liu, G.; Chen, J.; Guo, J.; Peng, Y. Org. Lett. 2015, $17,4870$.

[19] Hou, W.; Wei, Q.; Peng, Y. Adv. Synth. Catal. 2016, 358, 1035.

[20] (a) Smits, R.; Cadicamo, C. D.; Burger, K.; Koksch, B. Chem. Soc. Rev. 2008, 37, 1727.

(b) Mikami, K.; Itoh, Y.; Yamanaka, M. Chem. Rev. 2004, 104, 1.

[21] For some recent reviews, see:

(a) Zhou, Y.; Wang, J.; Gu, Z.; Wang, S.; Zhu, W.; Aceña, J. L.; Soloshonok, V. A.; Izawa, K.; Liu, H. Chem. Rev. 2016, $2,422$.

(b) Yang, X.; Wu, T.; Phipps, R. J.; Toste, F. D. Chem. Rev. 2015, 2, 826.

(c) Wang, J.; Sánchez-Roselló, M.; Aceña, J. L.; del Pozo, C.; Sorochinsky, A. E.; Fustero, S.; Soloshonok, V. A.; Liu, H. Chem. Rev. 2014, 114, 2432

[22] Huang, X.; Liu, M.; Jasinski, J. P.; Peng, B.; Zhang, W. Adv. Synth. Catal. 2017, 359, 1919.

[23] Wang, Y.; Yu, D. F.; Liu, Y. Z.; Wei, H.; Luo, Y. C.; Dixon, D. J.; Xu, P. F. Chem.-Eur. J. 2010, 16, 3922.

[24] Enders, D.; Urbanietz, G.; Cassens-Sasse, E.; Keess, S.; Raabe, G. Adv. Synth. Catal. 2012, 354, 1481

[25] Wu, M. Y.; He, W. W.; Liu, X. Y.; Tan, B. Angew. Chem., Int. Ed. 2015, 54, 9409.

[26] Malerich, J. P.; Hagihara, K.; Rawal, V. H. J. Am. Chem. Soc. 2008, $130,14416$.

[27] (a) Giacalone, F.; Gruttadauria, M.; Agrigento, P.; Noto, R. Chem. Soc. Rev. 2012, 41, 2406

(b) Banik, S. M.; Levina, A.; Hyde, A. M.; Jacobsen, E. N. Science 2017, 358, 761.

(c) Deng, Y. H.; Zhang, X. Z.; Yu, K. Y.; Yan, X.; Du, J. Y.; Huang, H.; Fan, C. A. Chem. Commun. 2016, 52, 4183.

(d) Bae, H. Y.; Song, C. E. ACS Catal. 2015, 5, 3613.

(e) Kasaplar, P.; Riente, P.; Hartmann, C.; Pericàs, M. A. Adv. Synth. Catal. 2012, 354, 2905. 
[28] (a) Didaskalou, C.; Kupai, J.; Cseri, L.; Barabas, J.; Vass, E.; Holtzl, T.; Szekely, G. ACS Catal. 2018, 8, 7430.

(b) Kucherenko, A. S.; Kostenko, A. A.; Komogortsev, A. N.; Lichitsky, B. V.; Fedotov, M. Y.; Zlotin, S. G. J. Org. Chem. 2019, 84, 4304.

(c) Liu, K.; Khan, I.; Cheng, J.; Hsueh, Y. J.; Zhang, Y. J. ACS Catal. 2018, 8, 11600 .

(d) Vural, U.; Durmaz, M.; Sirit, A. Org. Chem. Front. 2016, 3, 730 .

(e)Yang, W.; Du, D. M. Org. Lett. 2010, 12, 5450.

[29] For some selected reports and recent reviews, see:

(a) Shi, F.; Tao, Z. L.; Luo, S. W.; Tu, S. J.; Gong, L. Z. Chem.-Eur. J. 2012, 18, 6885 .

(b) Xi, N.; Arvedson, S.; Eisenberg, S.; Han, N.; Handley, M.; Huang, L.; Huang, Q.; Kiselyov, A.; Liu, Q.; Lu, Y.; Nunez, G.; Osslund, T.; Powers, D.; Tasker, A. S.; Wang, L.; Xiang, T.; Xu, S.; Zhang, J.; Zhu, J.; Kendall, R.; Dominguez, C. Bioorg. Med. Chem. Lett. 2004, 14, 2905.

(c) Periyasami, G.; Raghunathan, R.; Surendiran, G.; Mathivanan, N. Bioorg. Med. Chem. Lett. 2008, 18, 2342.

(d) Girgis, A. S. Eur. J. Med. Chem. 2009, 44, 91.

(e) Ali, M. A.; Ismail, R.; Choon, T. S.; Yoon, Y. K.; Wei, A. C.; Pandian, S.; Kumar, R. S.; Osman, H.; Manogaran, E. Bioorg. Med. Chem. Lett. 2010, 20, 7064.

(f) Hong, L.; Wang, R. Adv. Synth. Catal. 2013, 355, 1023.

(g) Tian, L.; Luo, Y. C.; Hu, X. Q.; Xu, P. F. Asian J. Org. Chem. 2016, 5, 580 .

(h) Mei, G. J.; Shi, F. Chem. Commun. 2018, 54, 6607.

(i) Fang, X.; Wang, C. J. Org. Biomol. Chem. 2018, 16, 2591.

(j) Cheng, D.; Ishihara, Y.; Tan, B.; Barbas III, C. F. ACS Catal. 2014, 4, 743 .

(k) Wang, Y. C.; Wang, J. L.; Burgess, K. S.; Zhang, J. W.; Zheng, Q. M.; Pu, Y. D.; Yan, L. J.; Chen, X. B. RSC Adv. 2018, 8, 5702.

(1) Zhang, D.; Qin, Y. Acta Chim. Sinica 2013, 71, 147 (in Chinese).

(张丹, 秦勇, 化学学报, 2013, 71, 147.)

(m) Zhang, L. L.; Zhang, J. W.; Xiang, S. H.; Guo, Z.; Tan, B. Org. Lett. 2018, 20, 6022 .

(n) Zhang, L. L.; Zhang, J. W.; Xiang, S. H.; Guo, Z.; Tan, B. Chin. J. Chem. 2018, 36, 1182 .

[30] Tian, L.; Hu, X. Q.; Li, Y. H.; Xu, P. F. Chem. Commun. 2013, 49, 7213.

[31] Chen, D. F.; Zhao, F.; Hu, Y.; Gong, L. Z. Angew. Chem., Int. Ed. 2014, 53, 10763.

[32] (a) Eschenbrenner-Lux, V.; Küchler, P.; Ziegler, S.; Kumar, K.; Waldmann, H. Angew. Chem., Int. Ed. 2014, 53, 2134.

(b) Mengozzi, L.; Gualandi, A.; Cozzi, P. G. Chem. Sci. 2014, 5, 3915 .

(c) Khashper, A.; Lubell, W. D. Org. Biomol. Chem. 2014, 12, 5052 .

(d) Friedman, R. K.; Rovis, T. J. Am. Chem. Soc. 2009, 131, 10775. (e) Perreault, S.; Rovis, T. Chem. Soc. Rev. 2009, 38, 3149.

[33] Blümel, M.; Chauhan, P.; Hahn, R.; Raabe, G.; Enders, D. Org. Lett. 2014, 16, 6012.

[34] Blüemel, M.; Chauhan, P.; Vermeeren, C.; Dreier, A.; Lehmann, C.; Enders, D. Synthesis 2015, 47, 3618.

[35] For recent reviews on the synthesis of tetrahydropyrans, see: (a) Yeung, K. S.; Paterson, I. Chem. Rev. 2005, 105, 4237.

(b) Nising, C. F.; Braese, S. Chem. Soc. Rev. 2012, 41, 988.

(c) Larrosa, I.; Romea, P.; Urpí. F. Tetrahedron 2008, 64, 2683.

[36] Hahn, R.; Raabe, G.; Enders, D. Org. Lett. 2014, 16, 3636.

[37] Chauhan, P.; Mahajan, S.; Loh, C. C.; Raabe, G.; Enders, D. Org. Lett. 2014, 16, 2954.

[38] Chauhan, P.; Urbanietz, G.; Raabe, G.; Enders, D. Chem. Commun. 2014, 50, 6853 .

[39] Chauhan, P.; Mahajan, S.; Raabe, G.; Enders, D. Chem. Commun. 2015, 51, 2270.

[40] Sun, Q. S.; Chen, X. Y.; Zhu, H.; Lin, H.; Sun, X. W.; Lin, G. Q. Org. Chem. Front. 2015, 2, 110.
[41] Sun, Q. S.; Zhu, H.; Lin, H.; Tan, Y.; Yang, X. D.; Sun, X. W.; Sun, X. Tetrahedron Lett. 2016, 57, 5768.

[42] For some typical references in this area, see: (a) Yang, J.; Liu, G. Y.; Lu, D. L.; Dai, F.; Qian, Y. P.; Jin, X. L.; Zhou, B. Chem.-Eur. J. 2010, 16, 12808.

(b) Koufaki, M.; Theodorou, E.; Galaris, D.; Nousis, L.; Katsanou, E. S.; Alexis, M. N. J. Med. Chem. 2006, 49, 300.

(c) Koufaki, M.; Detsi, A.; Theodorou, E.; Kiziridi, C.; Calogeropoulou, T.; Vassilopoulos, A.; Kourounakis, A. P.; Rekka, E.; Kourounakis, P. N.; Gaitanaki, C.; Papazafiri, P. Bioorg. Med. Chem. 2004, 12, 4835.

(d) Reddy, K. A.; Lohray, B. B.; Bhushan, V.; Reddy, A. S.; Mamidi, N. V. S. R.; Reddy, P. P.; Saibaba, V.; Reddy, N. J.; Suryaprakash, A.; Misra, P.; Vikramadithyan, R. K.; Rajagopalan, R. J. Med. Chem. 1999, 42, 3265.

[43] Xiao, Y.; Lin, J. B.; Zhao, Y. N.; Liu, J. Y.; Xu, P. F. Org. Lett. 2016, $18,6276$.

[44] (a) Dalpozzo, R.; Bartoli, G.; Bencivenni, G. Chem. Soc. Rev. 2012, $41,7247$.

(b) Zhang, J.; Qian, Z.; Wu, X.; Ding, Y.; Li, J.; Lu, C.; Shen, Y. Org. Lett. 2014, 16, 2752.

(c) Zhou, F.; Liu, Y. L.; Zhou, J. Adv. Synth. Catal. 2010, 352, 1381 .

(d) Ziarani, G. M.; Moradi, R.; Lashgari, N. Tetrahedron: Asymmetry 2015, 26, 517.

(e) Galliford, C. V.; Scheidt, K. A. Angew. Chem., Int. Ed. 2007, 46, 8748 .

(f) Shen, K.; Liu, X.; Lin, L.; Feng, X. Chem. Sci. 2012, 3, 327.

[45] Lian, X. L.; Meng, J.; Han, Z. Y. Org. Lett. 2016, 18, 4270.

[46] Urruzuno, I.; Mugica, O.; Oiarbide, M.; Palomo, C. Angew. Chem., Int. Ed. 2017, 56, 2059.

[47] (a) Hamama, W. S.; El-Gohary, H. G.; Kuhnert, N; Zoorob, H. H. Curr. Org. Chem. 2012, 16, 373.

(b) Horton, D. A.; Bourne, G. T.; Smythe, M. L. Chem. Rev. 2003, 103, 893.

(c) Yoshida, H.; Yanai, H.; Namiki, Y.; Fukatsu-Sasaki, K.; Furutani, N.; Tada, N. CNS Drug Rev. 2006, 12, 9.

(d) Hadi, V.; Koh, Y. H.; Sanchez, T. W.; Barrios, D.; Neamati, N.; Jung, K. W. Bioorg. Med. Chem. Lett. 2010, 20, 6854.

(e) Chande, M. S.; Barve, P. A.; Suryanarayan, V. J. Heterocycl. Chem. 2007, 44, 49.

[48] Li, J. H.; Cui, Z. H.; Du, D. M. Org. Chem. Front. 2016, 3, 1087.

[49] (a) Wang, T.; Yu, Z.; Hoon, D. L.; Huang, K. W.; Lan, Y.; Lu, Y. Chem. Sci. 2015, 6, 4912.

(b) Ibrahim, D. A. Eur. J. Med. Chem. 2009, 44, 2776.

(c) Abdel-Wahab, B. F.; Abdel-Latif, E.; Mohamed, H. A.; Awad, G. E. Eur. J. Med. Chem. 2012, 52, 263.

(d) Bayomi, S. M.; El-Kashef, H. A.; El-Ashmawy, M. B.; Nasr, M. N.; El-Sherbeny, M. A.; Abdel-Aziz, N. I.; El-Sayed, M. A. A.; Suddek, G. M.; El-Messery, S. M.; Ghaly, M. A. Eur. J. Med. Chem. 2015, 101, 584 .

(e) Ding, C. R.; Pan, Y. Y.; Yin, X.; Tan, C. X. Chin. J. Org. Chem. 2019, 39, 2099 (in Chinese).

(丁成荣, 潘亚运, 殷许, 谭成侠, 有机化学, 2019, 39, 2099.)

[50] Xiao, Y.; Jiang, R.; Wang, Y.; Zhou, Z. Adv. Synth. Catal. 2018, 360, 1961.

[51] For some typical references in this area, see:

(a) Maji, R.; Mallojjala, S. C.; Wheeler, S. E. Chem. Soc. Rev. 2018, 47, 1142 .

(b) Zhang, Y. L.; He, B. J.; Xie, Y. W.; Wang, Y. H.; Wang, Y. L.; Shen, Y. C.; Huang, Y. Y. Adv. Synth. Catal. 2019, 361, 3074.

(c) Kang, Q.; Zhao, Z. A.; You, S. L. J. Am. Chem. Soc. 2007, 129, 1484.

(d) Wang, S. G.; Yin, Q.; Zhuo, C. X.; You, S. L. Angew. Chem., Int. Ed. 2015, 54, 647

(e) Yin, Q.; Wang, S. G.; You, S. L. Org. Lett. 2013, 15, 2688.

(f) Zhang, K. F.; Nie, J.; Guo, R.; Zheng, Y.; Ma, J. A. Adv. Synth. Catal. 2013, 355, 3497. 
(g) Zhang, Y.; Ao, Y. F.; Huang, Z. T.; Wang, D. X.; Wang, M. X.; Zhu, J. Angew. Chem. Int. Ed. 2016, 55, 5282.

(h) Smith, M. J.; Reichl, K. D.; Escobar, R. A.; Heavey, T. J.; Coker, D. F.; Schaus, S. E.; Porco Jr, J. A. J. Am. Chem. Soc. 2018, 141,148 .

[52] (a) Akiyama, T.; Itoh, J.; Yokota, K.; Fuchibe, K. Angew. Chem., Int. Ed. 2004, 43, 1566

(b) Uraguchi, D.; Terada, M. J. Am. Chem. Soc. 2004, 126, 5356.

(c) Uraguchi, D.; Sorimachi, K.; Terada, M. J. Am. Chem. Soc. 2004, 126, 11804

[53] (a) Wang, Y. B.; Tan, B. Acc. Chem. Res. 2018, 51, 534.

(b) Xia, Z. L.; Zheng, C.; Xu, R. Q.; You, S. L. Nat. commun. 2019, 10,3150 .

(c) Zhang, L.; Xiang, S. H.; Wang, J. J.; Xiao, J.; Wang, J. Q.; Tan, B. Nat. Commun. 2019, 10, 566 .

(d) Li, J. S.; Liu, Y. J.; Li, S.; Ma, J. A. Chem. Commun. 2018, 54, 9151.

(e) Su, Y. J.; Shi, F. Q. Chin. J. Org. Chem. 2010, 30, 486 (in Chinese)

(苏亚军, 史福强, 有机化学, 2010, 30, 486.)

[54] Zhu, G.; Wang, B.; Bao, X.; Zhang, H.; Wei, Q.; Qu, J. Chem. Commun. 2015, 51, 15510.

[55] Wu, S.; Zhu, G.; Wei, S.; Chen, H.; Qu, J.; Wang, B. Org. Biomol.
Chem. 2018, 16, 807.

[56] For some recent reviews, see:

(a) Si, Y.; Chen, M.; Wu, L. Chem. Soc. Rev. 2016, 45, 690.

(b) Qi, J.; Lai, X.; Wang, J.; Tang, H.; Ren, H.; Yang, Y.; Jin, Q.; Zhang, L.; Yu, R.; Ma, G.; Su, Z.; Zhao, H.; Wang, D. Chem. Soc. Rev. 2015, 44, 6749 .

(c) Chen, Y.; Chen, H. R.; Shi, J. L. Acc. Chem. Res. 2014, 47, 125

(d) Sasidharan, M.; Nakashima, K. Acc. Chem. Res. 2014, 47, 157.

[57] Dai, F.; Zhao, Z.; Xie, G.; Feng, D.; Ma, X. ChemCatChem 2017, 9 , 89.

[58] (a) Lu, A.; Liu, T.; Wu, R.; Wang, Y.; Zhou, Z.; Wu, G.; Fang, J.; Tang, C. Eur. J. Org. Chem. 2010, 5777.

(b) Lu, A.; Liu, T.; Wu, R.; Wang, Y.; Wu, G.; Zhou, Z.; Fang, J.; Tang, C. J. Org. Chem. 2011, 76, 3872.

[59] Sun, J.; Jiang, C.; Zhou, Z. Eur. J. Org. Chem. 2016, 2016, 1165.

[60] (a) Dong, S.; Feng, X.; Liu, X. Chem. Soc. Rev. 2018, 47, 8525.

(b) Xie, L.; Dong, S.; Zhang, Q.; Feng, X.; Liu, X. Chem. Commun. 2018, 55, 87.

(c) Kang, T.; Zhao, P.; Yang, J.; Lin, L.; Feng, X.; Liu, X. Chem.-Eur. J. 2018, 24, 3703.

(d) Cui, X. Y.; Tan, C. H.; Leow, D. Org. Biomol. Chem. 2019, 17, 4689.

[61] Chen, Y.; Liu, X.; Luo, W.; Lin, L.; Feng, X. Synlett 2017, 28, 966.

(Li, L.; Fan, Y.) 